\title{
Anomaly of non-Abelian discrete symmetries
}

\author{
Tatsuo Kobayashi and Hikaru Uchida \\ Department of Physics, Hokkaido University, Sapporo 060-0810, Japan
}

(Received 28 November 2021; accepted 9 February 2022; published 28 February 2022)

\begin{abstract}
We study anomalies of non-Abelian discrete symmetries; which part of non-Abelian group is anomaly free and which part can be anomalous. It is found that the anomaly-free elements of the group $G$ generate a normal subgroup $G_{0}$ of $G$ and the residue class group $G / G_{0}$, which becomes the anomalous part of $G$, is isomorphic to a single cyclic group. The derived subgroup $D(G)$ of $G$ is useful to study the anomaly structure. This structure also constrains the structure of the anomaly-free subgroup; the derived subgroup $D(G)$ should be included in the anomaly-free subgroup. We study the detail structure of the anomaly-free subgroup from the structure of the derived subgroup in various discrete groups. For example, when $G=S_{n} \simeq A_{n} \rtimes Z_{2}$ and $G=\Delta\left(6 n^{2}\right) \simeq \Delta\left(3 n^{2}\right) \rtimes Z_{2}$, in particular, $A_{n}$ and $\Delta\left(3 n^{2}\right)$ are at least included in the anomaly-free subgroup, respectively. This result holds in any arbitrary representations.
\end{abstract}

DOI: 10.1103/PhysRevD.105.036018

\section{INTRODUCTION}

Symmetries are significant to understand various physical phenomena. The standard model of particle physics can explain the strong and electroweak interactions by the gauge $S U(3) \times S U(2) \times U(1)$ symmetry. Other continuous global and local symmetries are also important in particle physics, e.g., baryon and lepton number symmetries. In addition, Abelian and non-Abelian continuous symmetries are often used for model building.

Not only continuous symmetries, but also discrete symmetries are important. $C, P$, and $T$ are essential discrete symmetries in particle physics. The $R$ parity is useful to forbid the fast proton decay in minimal supersymmetric standard model. The $Z_{N}$ symmetry can be used to stabilize dark matter candidates. The origin of the quark and lepton flavor structures is one of the most significant mysteries in particle physics. Indeed, a lot of studies have been carried out. Among them one of most interesting approaches is to impose non-Abelian discrete flavor symmetries [1-6] on the generations of quarks and leptons.

However, even if there exists a symmetry in a theory at classical level, the symmetry can be broken by quantum effects, that is, the anomaly. In the case of continuous symmetry, global chiral $U(1)$ symmetry, for example, can be broken by the chiral anomaly unless the sum of $U(1)$ charges of chiral fermions vanishes. Fujikawa's method $[7,8]$ is a useful way to compute such chiral anomalies.

Published by the American Physical Society under the terms of the Creative Commons Attribution 4.0 International license. Further distribution of this work must maintain attribution to the author(s) and the published article's title, journal citation, and DOI. Funded by SCOAP ${ }^{3}$.
Similarly, anomalies of the Abelian discrete symmetry $Z_{N}$ were studied in [9-11]. Furthermore, anomalies of nonAbelian symmetries were studied by using the Fujikawa's method in Ref. [12]. Each element $g$ of the non-Abelian group $G$ generates the Abelian discrete symmetry $Z_{N}$ when $g^{N}=e$, where $e$ is the identity. If all of these Abelian symmetries corresponding to all the elements $g$ in $G$ are anomaly free, then the full symmetry $G$ is anomaly free. When some parts are anomalous, unbroken symmetry corresponds to the subgroup of $G$, which does not include anomalous elements. Indeed, Refs. [2,3] show which elements are anomaly free or can be anomalous for various examples of discrete groups. In addition, Ref. [13] shows the anomaly-free condition by introducing the derived subgroup of $G, D(G)$. Here, the derived subgroup is generated by commutator elements, $x y x^{-1} y^{-1}$, where $x$ and $y$ are elements of $G$. In particular, it shows that perfect groups, which are defined as $D(G)=G$, are perfectly anomaly free. In this paper, we study more detail anomaly structure of nonAbelian discrete symmetries. That is, we explore the detail structure of anomaly-free subgroups and anomalous parts of non-Abelian discrete symmetries generically.

This paper is organized as follows. In Sec. II, we review anomalies of (discrete) symmetry. In particular, a group element whose determinant is trivial becomes an anomalyfree transformation. In Sec. III, we study anomaly-free and anomalous structure of a discrete group, explored from the determinant of a representation. We have found that the anomaly-free elements construct a normal subgroup of the discrete group and the residue class group, which becomes the anomalous part, is isomorphic to a single cyclic group. This structure is important to explore the structure of the anomaly-free subgroup even if we do not 
specify the representation. The derived subgroup is the smallest normal subgroup, which leads the residue class group isomorphic to an Abelian group including a single cyclic group. This structure constrains the structure of the anomaly-free subgroup; the derived subgroup $D(G)$ should be included in the anomaly-free subgroup. Then, we study the detail structure of the anomaly-free subgroup through various examples of discrete groups in Sec. IV. In Sec. V, we comment on generic theories. In Sec. VI, we conclude this study. In Appendix A, the fundamental homomorphism and then the isomorphism theorems as well as the correspondence theorem are arranged. In Appendix B, some properties related to semidirect products are given. In Appendix C, some properties of finite Abelian groups are given.

\section{ANOMALIES OF DISCRETE SYMMETRY}

First, we briefly review anomalies of discrete symmetry [12]. Let us assume that a classical action $S$ with a set of chiral fermions $\psi_{L}=P_{L} \Psi$ is invariant under unitary transformation for the fermions, $\psi_{L} \rightarrow \rho(g) \psi_{L}, \forall g \in G$, where $G$ is a group and $\rho(g)$ is a unitary representation of $g \in G$. In this case, we say that the theory, at least at classical level, has chiral $G$ symmetry. For example, in the case of $G=Z_{N}$ symmetry, the generator $g \in Z_{N}$ satisfies $g^{N}=e$ and the unitary representation can be expressed as $\rho(g)_{j k}=e^{i \alpha q_{j}} \delta_{j k}$ with the phase parameter, $\alpha=2 \pi / N$, and the $Z_{N}$ charge of $j$ th component of $\psi_{L}, q_{j} \in \mathbb{Z} / N \mathbb{Z}$.

However, such a classical chiral symmetry can be broken at quantum level. First, let us see the global $G=Z_{N}$ symmetry under background non-Abelian gauge fields as well as gravity. We denote the gauge group by $G_{\text {gauge }}$ and the fermions have a representation $\mathbf{R}$ under $G_{\text {gauge. }}$ In the Fujikawa's method $[7,8]$, in particular, the measure in the path integral, $\int D \Psi D \bar{\Psi} e^{i S}$, can transform as

$$
D \Psi D \bar{\Psi} \rightarrow J(\alpha) D \Psi D \bar{\Psi},
$$

where the Jacobian can be written as $[14,15]$,

$$
J(\alpha)=\exp \left[i \int d^{4} x\left(A(x ; \alpha)_{\text {gauge }}+A(x ; \alpha)_{\text {grav }}\right)\right],
$$

with $\alpha=2 \pi / N$. The anomaly functions are written by

$$
A(x ; \alpha)_{\text {gauge }}=\frac{1}{32 \pi^{2}} \epsilon^{\mu \nu \rho \sigma} \operatorname{Tr}\left(\alpha q_{j}\left[F_{\mu \nu} F_{\rho \sigma}\right]\right),
$$

where $\operatorname{Tr}$ denotes the summation over all internal indices, and

$$
A(x ; \alpha)_{\text {grav }}=-\frac{1}{2} \frac{1}{384 \pi^{2}} \frac{1}{2} \epsilon^{\mu \nu \rho \sigma} R_{\mu \nu}^{\lambda \gamma} R_{\rho \sigma \lambda \gamma} \operatorname{tr}\left(\alpha q_{j} \mathbf{R}\right) .
$$

The index theorems [14,15] imply

$$
\begin{aligned}
& \int d^{4} x \frac{1}{32 \pi^{2}} \epsilon^{\mu \nu \rho \sigma} F_{\mu \nu}^{a} F_{\rho \sigma}^{b} \operatorname{tr}\left[t^{a} t^{b}\right] \in \mathbb{Z}, \\
& \frac{1}{2} \int d^{4} x \frac{1}{384 \pi^{2}} \frac{1}{2} \epsilon^{\mu \nu \rho \sigma} R_{\mu \nu}^{\lambda \gamma} R_{\rho \sigma \lambda \gamma} \in \mathbb{Z},
\end{aligned}
$$

where $t^{a, b}$ denote generators of $G_{\text {gauge }}$ in the $\mathbf{R}$ representation. We use the normalization of Dynkin index $T_{2}(\mathbf{R})$,

$$
T_{2}(\mathbf{R}) \delta_{a b}=\operatorname{tr}\left[t^{a} t^{b}\right],
$$

such that $T_{2}(\mathbf{R})=1 / 2$ for $N$ fundamental representation of $S U(N)$ and $T_{2}(\mathbf{R})=1$ for $2 N$ vector representation of $S O(2 N)$. For example, we have $T_{2}(\mathbf{R})=3$ for 27 representation of $E_{6}$. Thus, the anomaly-free condition for the mixed anomaly $Z_{N}-G_{\text {gauge }}-G_{\text {gauge }}$ is obtained as

$$
\begin{gathered}
J(\alpha)=e^{2 \pi i \sum q_{j} 2 T_{2}(\mathbf{R}) n / N}=1, \quad \forall n \in \mathbb{Z} \Leftrightarrow \\
\sum q_{j} 2 T_{2}(\mathbf{R}) \equiv 0 \quad(\bmod N) .
\end{gathered}
$$

Otherwise, the $Z_{N}$ symmetry can be anomalous.

Next, let us see the global non-Abelian discrete symmetry $G$. We can study its anomalies similarly as the $Z_{N}$ symmetry case since each element $g$ of the group $G$ satisfies $g^{N(g)}=e$, where $N(g)$ is the order of $g$. In general, fermions construct a multiplet under the non-Abelian symmetry $G$. For such a multiplet, the unitary representation of $g \in G$, $\rho(g)$, forms as unitary matrix. Here, we can always make the $\rho(g)$ diagonalized as $\rho(g)_{j k}=e^{i \alpha(g) q_{j}(g)} \delta_{j k}$ with the phase parameter of the $g$ transformation, $\alpha(g)=2 \pi / N(g)$, and the charge of $j$ th component of the multiplet for $g$ transformation, $q_{j} \in \mathbb{Z} / N(g) \mathbb{Z}$, by taking the appropriate base of the fermions. Note that, in such a base, the unitary matrices of some of the other elements $g^{\prime} \in G, \rho\left(g^{\prime}\right)$, become nondiagonalized matrices. Then, we can apply the analysis of the anomalies of the $Z_{N}$ symmetry to the anomalies of the non-Abelian discrete symmetry $G$. Suppose that fermion multiplets correspond to the representation $\rho(g)$ of the non-Abelian discrete symmetry $G$ and representations $\mathbf{R}$ of the non-Abelian gauge symmetry $G_{\text {gauge }}$. Then, the anomaly-free condition for the mixed anomalies $G-G_{\text {gauge }}-G_{\text {gauge }}$ is written by

$$
\begin{aligned}
J(\alpha(g)) & =e^{2 \pi i \sum q_{j} 2 T_{2}(\mathbf{R}) n / N(g)}=\left(e^{2 \pi i Q(g) / N(g)}\right)^{\sum_{\mathbf{R}} 2 T_{2}(\mathbf{R}) n} \\
& =(\operatorname{det} \rho(g))^{\sum_{\mathbf{R}} 2 T_{2}(\mathbf{R}) n}=1, \quad \forall n \in \mathbb{Z},
\end{aligned}
$$

where $Q(g) \equiv \sum_{j} q_{j}(g)$ and this $Q(g)$ is preserved even if the representation $\rho(g)$ is not diagonalized. Hence, we can say the symmetries including only the elements $g$ corresponding to

$$
\operatorname{det} \rho(g)=1,(\Leftrightarrow Q(g) \equiv 0(\bmod N(g))),
$$


are always anomaly free. Other parts in $G$ can be anomalous. The anomalies of symmetries corresponding to elements $g$ with $\rho(g) \neq 1$ depend on matter contents. That is, for $\sum_{\mathbf{R}} 2 T_{2}(\mathbf{R})=M$, the subgroup constructed by elements $g$ with $(\operatorname{det} \rho(g))^{M}=1$ is anomaly free, although the subgroup constructed by elements $g$ with $\operatorname{det} \rho(g)=1$ is always anomaly free. Thus, the determinant $\operatorname{det} \rho(g)$ is the key point in the analysis of following sections.

\section{ANOMALY STRUCTURE EXPLORED FROM DETERMINANT OF REPRESENTATIONS}

In this section, we study the anomaly structure by use of concrete representations $\rho(g)$ for elements $g$ in the non-Abelian symmetry $G$. Here, we concentrate mainly on the theory with $\sum_{\mathbf{R}} 2 T_{2}(\mathbf{R})=1$ and the anomaly-free condition $\operatorname{det} \rho(g)=1$. However, it is straightforward to extend our analysis to the theory with $\sum_{\mathbf{R}} 2 T_{2}(\mathbf{R})=$ $M>1$ and anomaly-free condition $(\operatorname{det} \rho(g))^{M}=1$.

Given representations of $\forall g \in G, \rho(g)$, we can calculate $\operatorname{det} \rho(g)$ explicitly. Suppose that $g^{N(g)}=e$ and then $\operatorname{det} \rho(g)^{N(g)}=1$ for a fixed element $g$, and also $(\operatorname{det} \rho(g))^{N}=1$ for any element $g$ in $G$. Then, we can write all of them as det $\rho(g)=e^{2 \pi i Q^{\prime}(g) / N}(\forall g \in G)$, where $Q^{\prime}(g)$ is given by $Q^{\prime}(g)=Q(g) N / N(g)$. As shown in the previous section, if $\operatorname{det} \rho(g)=1\left(Q^{\prime}(g) \equiv 0(\bmod N)\right)$, the element $g$ corresponds to anomaly-free transformation. Then, we define

$$
G_{0} \equiv\left\{g_{0} \in G \mid \operatorname{det} \rho\left(g_{0}\right)=1\right\},
$$

as the subset of $G$. From the following proof, we can find $G_{0}$ becomes a normal subgroup of $G, G_{0} \triangleleft G$. Thus, if anomalous transformations are fully broken by quantum effects, the symmetry $G$ is broken to the normal subgroup $G_{0}$ at quantum level.

Proof.-We can prove that $G_{0}$ is a subgroup of $G$, $G_{0} \subset G$, from (I) and then $G_{0}$ is also a normal subgroup of $G, G_{0} \triangleleft G$, from (II).

(I) When we take $\forall g_{0} \in G_{0}$ and $\forall g_{0}^{\prime} \in G_{0}$ $\left(\operatorname{det} \rho\left(g_{0}\right)=\operatorname{det} \rho\left(g_{0}^{\prime}\right)=1\right.$ ), the element $g_{0} g_{0}^{\prime}$ is also included in $G_{0}, g_{0} g_{0}^{\prime} \in G_{0} \quad\left[\operatorname{det} \rho\left(g_{0} g_{0}^{\prime}\right)=1\right]$. In particular, the identity element $e$ is included in $G_{0}$ [det $\rho(e)=1$ ], and also when we take $\forall g_{0} \in G_{0}$ [det $\rho\left(g_{0}\right)=1$ ], the inverse element $g_{0}^{-1}$ is included in $G_{0}\left(\operatorname{det} \rho\left(g_{0}^{-1}\right)=1\right)$.

(II) When we take $\forall g_{0} \in G_{0} \quad\left(\operatorname{det} \rho\left(g_{0}\right)=1\right)$ and $\forall g \in G$, the conjugate element $g g_{0} g^{-1}$ is also included in $G_{0}, \quad g g_{0} g^{-1} \in G_{0} \quad\left(\operatorname{det} \rho\left(g g_{0} g^{-1}\right)=\right.$ $\left.\operatorname{det} \rho\left(g_{0}\right)=1\right)$.

Now, we can rewrite $\forall g \in G$, which satisfies $\operatorname{det} \rho(g)=$ $e^{2 \pi i k / N}\left(Q^{\prime}(g) \equiv k(\bmod N)\right)$ as $g=g_{0} g_{1}^{k}$, where $g_{1}$ satisfies $\operatorname{det} \rho\left(g_{1}\right)=e^{2 \pi i / N}\left(Q^{\prime}\left(g_{1}\right) \equiv 1(\bmod N)\right)$ and $\exists g_{0} \in G_{0}$.

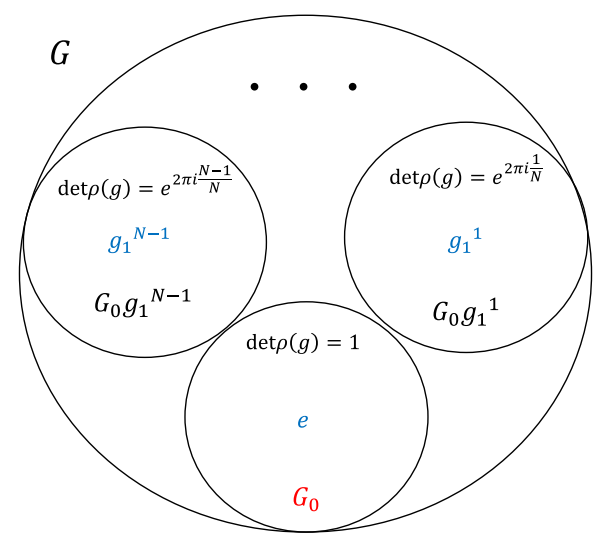

FIG. 1. Image of cosets $G_{0} g_{1}^{k}$ whose elements $g$ satisfy $\operatorname{det} \rho(g)=e^{2 \pi i k / N}$. The representative element of each coset is written by blue.

In other words, the coset, whose element $g$ satisfies $\operatorname{det} \rho(g)=e^{2 \pi i k / N}$, can be expressed as $G_{0} g_{1}^{k}$. (See also Fig. 1.) Actually, from the following proof, we can find such cosets consist the residue class group $G / G_{0} \simeq Z_{N}$.

Proof.-We can prove that the residue class group $G / G_{0}$ is Abelian from (I) and also isomorphic to $Z_{N}$ from (II).

(I) $G_{0} g_{1}^{k_{1}}$ and $G_{0} g_{1}^{k_{2}}$ satisfy the relation $\left(G_{0} g_{1}^{k_{1}}\right)\left(G_{0} g_{1}^{k_{2}}\right)=\left(G_{0} g_{1}^{k_{2}}\right)\left(G_{0} g_{1}^{k_{1}}\right)=G_{0} g_{1}^{k_{1}+k_{2}}$.

(II) $G_{0} g_{1}^{N-k}$ becomes inverse coset of $G_{0} g_{1}^{k}$ since $g_{1}^{N} \in$ $G_{0}\left(\operatorname{det} \rho\left(g_{1}^{N}\right)=1\right)$.

Here, the element $g_{1} \in G$ generally satisfies $g_{1}^{N}=g_{0}$, $\exists g_{0} \in G$ while it satisfies $g_{1}^{N\left(g_{1}\right)}=e$. Then, we find $G_{0} \cap$ $Z_{N\left(g_{1}\right)}=Z_{N\left(g_{1}\right) / N}$ in general, where $Z_{N\left(g_{1}\right)}$ is the subgroup of $G$ generated by $g_{1}$ and $Z_{N\left(g_{1}\right) / N}$ is the subgroup of $G_{0}$ generated by $g_{1}^{N}$. Actually, by using the isomorphism theorem 2 in Appendix A, we can also obtain

$$
G / G_{0} \simeq G_{0} Z_{N\left(g_{1}\right)} / G_{0} \simeq Z_{N\left(g_{1}\right)} / Z_{N\left(g_{1}\right) / N} \simeq Z_{N} .
$$

If $g_{1}$ satisfies $g_{1}^{N}=e \in G_{0}\left(N\left(g_{1}\right)=N\right)$, then, in particular, $g_{1}$ generates $Z_{N}$ subgroup of $G$ and the $Z_{N}$ subgroup satisfies $G=G_{0} Z_{N}$ and $G_{0} \cap Z_{N}=\{e\}$. Thus, in this case, $G$ can be decomposed ${ }^{1}$ as

$$
G \simeq G_{0} \rtimes Z_{N} .
$$

It means that the anomaly-free and anomalous parts of $G$ can be separated. In more general, if there exists $\exists g \in G$, which satisfies $N(g)=N$ and $\operatorname{gcd}(Q(g), N(g))=1, G$ can be expressed as Eq. (11) since this $g$ generates $Z_{N}$ subgroup of $G$ and it satisfies $G=G_{0} Z_{N}$ and $G_{0} \cap Z_{N}=\{e\}$. In particular, when $N$ is a prime number, they are automatically satisfied.

\footnotetext{
${ }^{1}$ See also Appendix B.
} 
Let us summarize the important points in this section again.

(i) Generally, the anomaly-free subset of $G, G_{0}$, becomes a normal subgroup of $G, G_{0} \triangleleft G$, and then the anomalous part becomes $G / G_{0} \simeq Z_{N}$, where $\operatorname{det} \rho(g)(\forall g \in G)$ can be expressed as $\operatorname{det} \rho(g)=e^{2 \pi i Q^{\prime}(g) / N}$.

(ii) In particular, if there exists $\exists g \in G$ which satisfies $N(g)=N$ and $\operatorname{gcd}(Q(g), N(g))=1, G$ can be expressed as $G \simeq G_{0} \rtimes Z_{N}$.

\section{DERIVED SUBGROUP}

In the previous section, by use of the representation of $\forall g \in G, \rho(g)$, we have shown $G / G_{0} \simeq Z_{N}$ generally. However, even without use of explicit representations, this result is still valid although we do not know the explicit number $N$ from the beginning. In this section, we study the detail structure of $G_{0}$ and $G / G_{0} \simeq Z_{N}$ from the structure of $G$. We note that the analysis in this section can be applied for any representations although we often consider a specific case.

First of all, we introduce the derived subgroup $D(G)$ of $G$,

$$
D(G) \equiv\left\langle x y x^{-1} y^{-1} \in G \mid x, y \in G\right\rangle,
$$

which is also called the commutator subgroup. The derived subgroup $D(G)$ is a normal subgroup $G, D(G) \triangleleft G$, which can be shown as

$$
\begin{aligned}
g\left(x y x^{-1} y^{-1}\right) g^{-1}= & \left(g x g^{-1}\right)\left(g y g^{-1}\right) \\
& \times\left(g x g^{-1}\right)^{-1}\left(g y g^{-1}\right)^{-1} \in D(G),
\end{aligned}
$$

for $\forall x y x^{-1} y^{-1} \in D(G)$ and $\forall g \in G$. The quotient $G / D(G)$ is Abelian, which means that any cosets $D(G) X \quad$ and $\quad D(G) Y \quad$ with $\quad X, Y \notin D(G)$ satisfy $(D(G) X)(D(G) Y)=(D(G) Y)(D(G) X)$, because of $X Y X^{-1} Y^{-1} \in D(G)$. Indeed, the derived subgroup $D(G)$ is smallest among normal subgroups $K_{G}$ of $G$ such that $G / K_{G}$ is Abelian. $^{2}$ Thus, we can find that

$$
G_{0} \supseteq D(G),
$$

because of $G / G_{0} \simeq Z_{N}$. We can actually check it as

$$
\operatorname{det} \rho\left(x y x^{-1} y^{-1}\right)=\operatorname{det}\left[\rho(x) \rho(y) \rho(x)^{-1} \rho(y)^{-1}\right]=1 .
$$

Therefore, the derived subgroup $D(G)$ of $G$ is always anomaly free. The whole anomaly-free subgroup $G_{0}$ is either the same as $D(G)$ or larger than $D(G)$. We study how large $G_{0}$ is compared with $D(G)$ in what follows.

\footnotetext{
${ }^{2}$ If $G$ itself is an Abelian group, then $D(G)=\{e\}$ is satisfied.
}

The order of $G / D(G)$ can be written as a product of prime numbers $p_{i},|G / D(G)|=\prod_{i=1}^{r} p_{i}^{A_{i}}=p_{1}^{A_{1}} \cdots p_{r}^{A_{r}}$. Then, by the fundamental structure theorem ${ }^{3}$ of finite Abelian group, $G / D(G)$ can be generally expressed as

$$
\begin{aligned}
G / D(G) \simeq & \left(Z_{p_{1}^{a_{1,1}}} \times \cdots \times Z_{p_{1}^{a_{1, n_{1}}}}\right) \\
& \times \cdots \times\left(Z_{p_{r}^{a_{r}, 1}} \times \cdots \times Z_{p_{r}^{a_{r}, n_{r}}}\right),
\end{aligned}
$$

where $a_{i, j}$ satisfy

$$
A_{i}=\sum_{j=1}^{n_{i}} a_{i, j}, \quad a_{i, j} \geq a_{i, j+1} .
$$

On the other hand, $G / G_{0}$ is isomorphic to a single cyclic group $Z_{N}$. Thus, the structure of the Abelian group $G / D(G)$ in Eq. (15) is important to study how large $G_{0}$ is compared with $D(G)$.

Now, let us classify our particle theories by the determinant of the representation (including reducible representation) of the element $X_{i, j}$ of $Z_{p_{i}}^{a_{i, j}}$ in the Abelian group $G / D(G),{ }^{4} \operatorname{det} \rho\left(X_{i, j}\right)$.

(i) We consider the theory in which the element $\forall X_{i, j} \in Z_{p_{i}^{a_{i, j}}}$ for $\forall i, j$ satisfies

$$
\operatorname{det} \rho\left(X_{i, j}\right)=1 .
$$

In this case, we can easily find that

$$
G_{0}=G
$$

(ii) We consider the theory in which the element $\exists X_{i, j} \in Z_{p_{i} a_{i, j}}-\{e\}$ for $\exists i, j$ satisfies

$$
\operatorname{det} \rho\left(X_{i, j}\right)=1 \text {, }
$$

while any other element $\forall X_{i, j}^{\prime} \in Z_{p_{i} a_{i, j}}-\left\{e, X_{i, j}\right\}$ for $\forall i, j$ satisfies

$$
\operatorname{det} \rho\left(X_{i, j}^{\prime}\right) \neq 1
$$

In this case, we can find that

$$
G \supset G_{0} \supset D(G) .
$$

Although we do not discuss the detail of this class in this paper since there are several patterns of this type

\footnotetext{
${ }^{3}$ See also Appendix C.

${ }^{4}$ Here, $Z_{p}{ }^{a_{i, j}}$ is a subgroup of the Abelian group $G / D(G)$ but not a subgroup of $G$ in general. Then, the element $X_{i, j} \in Z_{p_{i}}^{a_{i, j}}$ corresponds to an element of the coset $D(G) X_{i, j}$ in $G$. However, since it is satisfied that $\operatorname{det} \rho\left(g_{D}\right)=1 \forall g_{D} \in D(G)$, the determinant $\operatorname{det} \rho\left(X_{i, j}\right)$ reflects on also $G$ as the same way.
} 
in some discrete groups $G$, one can also consider this class similarly as the following analysis.

(iii) We consider the theory in which the element $\forall X_{i, j} \in Z_{p_{i} a_{i, j}}-\{e\}$ for $\forall i, j$ satisfies

$$
\operatorname{det} \rho\left(X_{i, j}\right) \neq 1 \text {. }
$$

If there exists $a_{i, 2} \neq 0$ for $\exists i$, then $p_{i}^{a_{i, 2}}-1$ numbers of combination elements $X_{i, 1}^{m_{1}} X_{i, 2}^{m_{2}}$ satisfy

$$
\operatorname{det} \rho\left(X_{i, 1}^{m_{1}} X_{i, 2}^{m_{2}}\right)=1 \text {, }
$$

where $m_{1}$ and $m_{2}$ satisfy the following relation:

$$
\begin{aligned}
Q\left(X_{i, 1}^{m_{1}} X_{i, 2}^{m_{2}}\right) & =m_{1} Q\left(X_{i, 1}\right)+m_{2} Q\left(X_{i, 2}\right) p_{i}^{a_{i, 1}-a_{i, 2}} \equiv 0 \quad\left(\bmod p_{i}^{a_{i, 1}}\right), \\
\Leftrightarrow m_{1} Q\left(X_{i, 1}\right) & =p_{i}^{a_{i, 1}-a_{i, 2}} n, \quad m_{2} Q\left(X_{i, 2}\right)=p_{i}^{a_{i, 2}}-n, \quad \forall n \in \mathbb{Z} / p_{i}^{a_{i, 2}} \mathbb{Z}-\{0\} .
\end{aligned}
$$

Then, those $X_{i, 1}^{m_{1}} X_{i, 2}^{m_{2}}$ as well as $e$ are also included in $G_{0}$, which means

$$
G \supset G_{0} \supset D(G),
$$

and also they construct $Z_{p_{i}^{a_{i, 2}}}$ subgroup of $G_{0} / D(G)$. Note that, in general, if there are $Z_{N_{1}}$ and $Z_{N_{2}}$ symmetries in $G / D(G)$, where $N_{1}$ and $N_{2}$ are not coprime to each other, $\operatorname{gcd}\left(N_{1}, N_{2}\right)-1$ numbers of elements $X_{1}^{m_{1}} X_{2}^{m_{2}}\left(X_{1} \in\right.$ $\left.Z_{N_{1}}, X_{2} \in Z_{N_{2}}\right)$ as well as $e$ satisfy $\operatorname{det} \rho\left(X_{1} X_{2}\right)=1$ and then they construct $Z_{\operatorname{gcd}\left(N_{1}, N_{2}\right)}$ subgroup of $G_{0} / D(G)$. In a similar way, we can find that

$$
\begin{aligned}
G_{0} / D(G) \simeq & \left(Z_{p_{1}^{a_{1,2}}} \times \cdots \times Z_{p_{1}^{a_{1, n_{1}}}}\right) \times \cdots \\
& \times\left(Z_{p_{r}^{a_{r}, 2}} \times \cdots \times Z_{p_{r} a_{r, n_{r}}}\right) .
\end{aligned}
$$

Then, by using the isomorphism Theorem 3 in Appendix A and Eqs. (15) and (25), we can obtain

$Z_{N} \simeq G / G_{0} \simeq(G / D(G)) /\left(G_{0} / D(G)\right) \simeq Z_{\left(p_{1}^{a_{1,1}} \ldots p_{r}^{a_{r, 1}}\right)}$.

Indeed, when Eq. (21) is satisfied, the determinant $\operatorname{det} \rho(g)$ for $\forall g \in G$ can be expressed as $\operatorname{det} \rho(g)=$ $e^{2 \pi i Q^{\prime}(g) /\left(p_{1}^{a_{1}, \ldots} \ldots p_{r}^{a_{r}, 1}\right)}$, which means that we get Eq. (26). Note that $N=p_{1}^{a_{1,1}} \cdots p_{r}^{a_{r, 1}}=\prod_{i=1}^{r} p_{i}^{a_{i, 1}}$ is the least common multiple of orders of each $Z_{p_{i} a_{i, j}}$ in $G / D(G)$ and it becomes the maximum order of the anomalous $G / G_{0} \simeq Z_{N}$. In other words, the maximum order of the anomalous $G / G_{0} \simeq Z_{N}$ can be found by $G / D(G)$, which is determined by $G$.

In particular, if and only if it is also satisfied that $A_{i}=a_{i, 1}\left(a_{i, 2}=0\right)$ for all $i$, any element $X$ in the Abelian group $G / D(G)$ leads to

$$
\operatorname{det} \rho(X) \neq 1,
$$

which means that

$$
G_{0}=D(G),
$$

and then, by using Eq. (C3) in Appendix C, we can obtain

$$
Z_{N} \simeq G / G_{0}=G / D(G) \simeq Z_{\left(p_{1}^{A_{1}} \ldots p_{r}^{A_{r}}\right)} .
$$

Therefore, $D(G)$ gives us an important clue to obtain information about $G_{0}$ and $G / G_{0} \simeq Z_{N}$. In the following analysis, we mainly discuss this class (iii) that Eq. (21) is satisfied.

\section{A. Various examples of $G$}

Now, in this subsection, let us see the detail structure of $G_{0}$ and $G / G_{0} \simeq Z_{N}$ from the structure of $D(G)$ and $G / D(G)$ through specific examples.

First, when $G$ is a perfect group, defined as a group satisfying $D(G)=G$, obviously we can find that $G=G_{0}=D(G)$. In particular, non-Abelian simple groups such as $A_{n}(n \geq 5)$ and $\operatorname{PSL}\left(2, Z_{p}\right)(p \neq 2,3, p \in \mathbb{P})$ are the simplest examples of perfect groups. That is, the whole symmetry $G$ is always anomaly free. (See also Ref. [13].) Here, we mention about a simple group. The simple group is defined as the group $G$, such that it does not have any normal subgroups but $\{e\}$ and $G$ itself. Then, since $G / D(G)$ is Abelian, $D(G)$ must be equal to $G$ itself for a non-Abelian simple group.

On the other hand, an Abelian simple group, which is just isomorphic to $Z_{p}(p \in \mathbb{P})$, is not a perfect group since $D(G)=\{e\}$. In this case, the flavor model corresponds to either class (i), $G_{0}=G \simeq Z_{p}$, or the class (iii), $G_{0}=D(G)=\{e\}, G / G_{0} \simeq Z_{N}=Z_{p}$. The group $G=$ $A_{3} \simeq Z_{3}$ is an example of this case.

Next, let us consider the group $G$, which can be decomposed by a semidirect product, i.e., $G \simeq K_{G} \rtimes G^{(1)}$. We discuss this in the following four steps.

Step $1\left(G \simeq Z_{A} \rtimes Z_{B}\right) \quad$ Let us start with the simplest group, $G \simeq Z_{A} \rtimes Z_{B}\left(G / Z_{A} \simeq Z_{B}\right)$, where $A \geq 3$. Then, it is found that $D(G) \subseteq Z_{A}$. When we take $\alpha \in Z_{A}$ and $\beta \in Z_{B}$, they satisfy the following algebraic relations:

$$
\alpha^{A}=\beta^{B}=e
$$

and also 


$$
\begin{gathered}
\beta \alpha \beta^{-1}=\alpha^{m} \in Z_{A}, \quad\left(\beta \alpha \beta^{-1} \alpha^{-1}=\alpha^{m-1} \in D(G)\right), \quad m \in \mathbb{Z} / A \mathbb{Z}-\{0,1\}, \\
\Rightarrow \beta^{b} \alpha^{a} \beta^{-b}=a^{a m^{b}}, \quad\left(\beta^{b} \alpha^{a} \beta^{-b} \alpha^{-a}=a^{a\left(m^{b}-1\right)} \in D(G)\right), \quad a \in \mathbb{Z} / A \mathbb{Z}, \quad b \in \mathbb{Z} / B \mathbb{Z},
\end{gathered}
$$

where $m$ satisfies $^{5}$ the following conditions,

$$
\begin{aligned}
& \left(m^{b}-1\right)=(m-1)\left(\sum_{r=0}^{b-1} m^{r}\right) \not \equiv 0 \quad(\bmod A) \text { for } \forall b \\
& \left(m^{B}-1\right)=(m-1)\left(\sum_{r=0}^{B-1} m^{r}\right) \equiv 0(\bmod A) .
\end{aligned}
$$

From Eq. (32), we find that

$$
\begin{aligned}
D(G) & =\left\{\alpha^{a^{\prime} \operatorname{gcd}(m-1, A)} \mid a^{\prime} \in \mathbb{Z} /(A / \operatorname{gcd}(m-1, A)) \mathbb{Z}\right\} \\
& =Z_{A / \operatorname{gcd}(m-1, A)} \subseteq G_{0},
\end{aligned}
$$

while $D(G)=\{e\}$ in the case of $G \simeq Z_{A}$. Then, we find that

$$
G / D(G) \simeq Z_{\operatorname{gcd}(m-1, A)} \times Z_{B}
$$

since cosets $D(G) \alpha$ and $D(G) \beta$ satisfy

$$
\begin{aligned}
(D(G) \alpha)^{\operatorname{gcd}(m-1, A)} & =(D(G) \beta)^{B}=D(G), \\
(D(G) \beta)(D(G) \alpha)(D(G) \beta)^{-1} & =D(G)\left(\beta \alpha \beta^{-1} \alpha^{-1}\right) \alpha \\
& =D(G) \alpha .
\end{aligned}
$$

The above result can also be understood in terms of $Z_{A}$ charge constraint. Let us assume that the chiral fermions are $Z_{A}$ eigenstates, $\rho(\alpha)_{j k}=e^{2 \pi i q_{j} / A} \delta_{j k}$, where $q_{j}$ is the $Z_{A}$ charge of $j$ th component. Equation (31) shows that there exists a state with charge $m q_{j}$ and $\beta$ transforms the $j$ th component to the state with $m q_{j}$. Then, when we consider a fundamental irreducible representation, it becomes $B$-dimensional representation with $Z_{A}$ charge ${ }^{t}\left(q_{1}, q_{2}, q_{3}, \ldots, q_{B}\right)={ }^{t}(q, m q$, $\left.m^{2} q, \ldots, m^{B-1} q\right)$. It means that $Z_{A}$ charges in a multiplet are constrained by the semidirect product by $Z_{B}$, while there is no constraint for $Z_{B}$ charges. Thus, we obtain

$$
\operatorname{det} \rho(\alpha)=e^{2 \pi i \sum_{r=0}^{B-1} m^{r} q / A}=e^{2 \pi i q n / \operatorname{gcd}(m-1, A)},
$$

which means Eqs. (34) and (35).

Now, as discussed in the above general analysis, let us study the class (iii). Hereafter, we use $A^{\prime} \equiv \operatorname{gcd}(m-1, A)$. Then, the elements $\alpha^{x} \beta^{y}$, which satisfy

$$
\begin{aligned}
& Q\left(\alpha^{x}\right) B / \operatorname{gcd}\left(A^{\prime}, B\right)+Q\left(\beta^{y}\right) A^{\prime} / \operatorname{gcd}\left(A^{\prime}, B\right) \equiv 0 \quad\left(\bmod \operatorname{lcm}\left(A^{\prime}, B\right)\right) \\
\Leftrightarrow & x Q(\alpha)=\left(\operatorname{gcd}\left(A^{\prime}, B\right)-s\right) A^{\prime} / \operatorname{gcd}\left(A^{\prime}, B\right), y Q(\beta)=s B / \operatorname{gcd}\left(A^{\prime}, B\right), s \in \mathbb{Z} / \operatorname{gcd}\left(A^{\prime}, B\right) \mathbb{Z},
\end{aligned}
$$

also satisfy $\operatorname{det} \rho\left(\alpha^{x} \beta^{y}\right)=1$; that is, the elements of $\operatorname{gcd}\left(A^{\prime}, B\right)$ numbers of cosets $D(G) \alpha^{x} \beta^{y} \quad$ [including $\left.D(G) \alpha^{A^{\prime}}=D(G)\right]$ become the elements of $G_{0}$. In addition, since these cosets satisfy $\left(D(G) \alpha^{x} \beta^{y}\right)^{\operatorname{gcd}\left(A^{\prime}, B\right)}=D(G)$, they construct the (normal) subgroup of $G / D(G)$,

$$
G_{0} / D(G) \simeq Z_{\operatorname{gcd}\left(A^{\prime}, B\right)}
$$

On the other hand, the generators of $G_{0}$ are $\alpha^{A^{\prime}}$ and $\alpha^{x} \beta^{y}$, where $x, y$ satisfy the above conditions in Eq. (37), and they satisfy

\footnotetext{
${ }^{5}$ If $m=1$, then the group $G$ reduces to $G \simeq Z_{A} \times Z_{B}$. This case is the specific case in the above general analysis.
}

$$
\begin{aligned}
\left(\alpha^{A^{\prime}}\right)^{A / A^{\prime}} & =e,\left(\alpha^{x} \beta^{y}\right)^{\operatorname{gcd}\left(A^{\prime}, B\right)}=\left(\alpha^{A^{\prime}}\right)^{k=\frac{x\left(m^{y g c d}\left(A^{\prime}, B\right)\right.}{A^{\prime}\left(m^{y}-1\right)}}, \\
\left(\alpha^{x} \beta^{y}\right)\left(\alpha^{A^{\prime}}\right)\left(\alpha^{x} \beta^{y}\right)^{-1} & =\left(\alpha^{A^{\prime}}\right)^{m^{y}} .
\end{aligned}
$$

In particular, if $k \equiv 0\left(\bmod A / A^{\prime}\right)$, which means $\alpha^{x} \beta^{y}$ actually generates this $Z_{\mathrm{gcd}\left(A^{\prime}, B\right)}$ subgroup of $G_{0}$, then $G_{0}$ can be written as $G_{0} \simeq D(G) \rtimes Z_{\operatorname{gcd}\left(A^{\prime}, B\right)}=Z_{A / A^{\prime}} \rtimes$ $Z_{\operatorname{gcd}\left(A^{\prime}, B\right)}$. Then, by using the isomorphism Theorem 3 in Appendix A, we can obtain

$$
\begin{aligned}
Z_{N} & \simeq G / G_{0} \simeq(G / D(G)) /\left(G_{0} / D(G)\right) \\
& \simeq\left(Z_{A^{\prime}} \times Z_{B}\right) / Z_{\operatorname{gcd}\left(A^{\prime}, B\right)} \simeq Z_{\mathrm{lcm}\left(A^{\prime}, B\right)}
\end{aligned}
$$

where we also use Eq. (C4) in Appendix C for Eq. (35),

$$
G / D(G) \simeq Z_{A^{\prime}} \times Z_{B} \simeq Z_{\mathrm{gcd}\left(A^{\prime}, B\right)} \times Z_{\mathrm{lcm}(A, B)} .
$$


Similar to Eq. (37), there exists $\alpha^{x^{\prime}} \beta^{y^{\prime}}$ with $\exists\left(x^{\prime}, y^{\prime}\right)$, which satisfies

$$
\begin{aligned}
& Q\left(\alpha^{z^{\prime}}\right) B / \operatorname{gcd}\left(A^{\prime}, B\right)+Q\left(\beta^{y^{\prime}}\right) A^{\prime} / \operatorname{gcd}\left(A^{\prime}, B\right) \equiv 1 \\
& \quad\left(\bmod \operatorname{ccm}\left(A^{\prime}, B\right)\right),
\end{aligned}
$$

since $A^{\prime} / \operatorname{gcd}\left(A^{\prime}, B\right)$ and $B / \operatorname{gcd}\left(A^{\prime}, B\right)$ are coprime to each other. It means that this element $\alpha^{x^{\prime}} \beta^{y^{\prime}}$ corresponds to $g_{1}$ in the previous section. In addition, the coset $D(G) \alpha^{x^{\prime}} \beta^{y^{\prime}} \subset G_{0} \alpha^{x^{\prime}} \beta^{y^{\prime}} \quad$ satisfies $\quad\left(D(G) \alpha^{x^{\prime}} \beta^{y^{\prime}}\right)^{\operatorname{lcm}\left(A^{\prime}, B\right)}=$ $D(G) \subset G_{0}$. Then, similar to Eq. (39), this element generally satisfies

$$
\begin{aligned}
\left(\alpha^{x^{\prime}} \beta^{y^{\prime}}\right)^{\operatorname{lcm}\left(A^{\prime}, B\right)}=\left(\alpha^{A^{\prime}}\right)^{k^{\prime}=\frac{x^{\prime}\left(m^{y^{\prime}} \operatorname{lcm}\left(A^{\prime}, B\right)-1\right)}{A^{\prime}\left(m^{\prime}-1\right)}}, \\
\left(\alpha^{x^{\prime}} \beta^{y^{\prime}}\right)\left(\alpha^{A^{\prime}}\right)\left(\alpha^{x^{\prime}} \beta^{y^{\prime}}\right)^{-1}=\left(\alpha^{A^{\prime}}\right)^{m^{y^{\prime}}}, \\
\left(\alpha^{x^{\prime}} \beta^{y^{\prime}}\right)\left(\alpha^{x} \beta^{y}\right)\left(\alpha^{x^{\prime}} \beta^{y^{\prime}}\right)^{-1}=\left(\alpha^{A^{\prime}}\right)^{\ell^{\prime}=\left[x\left(m^{y^{\prime}}-1\right)-x^{\prime}\left(m^{y}-1\right)\right] / A^{\prime}}\left(\alpha^{x} \beta^{y}\right) .
\end{aligned}
$$

If $k^{\prime} \equiv 0\left(\bmod A / A^{\prime}\right)$, which means $\alpha^{x^{\prime}} \beta^{y^{\prime}}$ actually generates this $Z_{\mathrm{lcm}\left(A^{\prime}, B\right)}$ subgroup of $G$, then $G$ can be similarly written as $G \simeq G_{0} \rtimes Z_{\mathrm{lcm}\left(A^{\prime}, B\right)}$. In addition, if $\ell^{\prime} \equiv 0\left(\bmod A / A^{\prime}\right)$,

then the element $\alpha^{x^{\prime}} \beta^{y^{\prime}}$ commutes $\alpha^{x} \beta^{y}$. Thus, if $k, k^{\prime}, \ell^{\prime}$ are multiples of $A / A^{\prime}$, which means $Z_{\operatorname{gcd}\left(A^{\prime}, B\right)} \times Z_{\mathrm{lcm}(A, B)}$ in Eq. (41) [as well as each of $Z_{\operatorname{gcd}\left(A^{\prime}, B\right)}$ and $Z_{\operatorname{lcm}(A, B)}$ is actually a subgroup of $G$, then $G$ can be written as

$$
\begin{aligned}
G & \simeq D(G) \rtimes\left(Z_{\operatorname{gcd}\left(A^{\prime}, B\right)} \times Z_{\operatorname{lcm}(A, B)}\right) \simeq Z_{A / A^{\prime}} \rtimes\left(Z_{A^{\prime}} \times Z_{B}\right), \\
& \simeq\left(D(G) \rtimes Z_{\operatorname{gcd}\left(A^{\prime}, B\right)}\right) \rtimes Z_{\operatorname{lcm}(A, B)}, \\
& \simeq G_{0} \rtimes Z_{\operatorname{lcm}(A, B)},
\end{aligned}
$$

where the second line can be understood by Eqs. (B4) and (B5) in Appendix B.

Now, let us see examples. First, if $A=p \in \mathbb{P}$, we obtain $A^{\prime}=1$. Then, it is found $D(G)=Z_{A} \subseteq G_{0}$. When we consider the class (iii), we obtain $G_{0}=D(G)=Z_{A}$ and $G / G_{0} \simeq Z_{N}=Z_{B}$.

Second, let us study $G=D_{A} \simeq Z_{A} \rtimes Z_{2}$. In this case, we obtain $m=A-1$ from Eq. (33), and then we can find that

$$
D(G)= \begin{cases}<\tilde{\alpha} \mid \tilde{\alpha}^{A / 2}=e>=Z_{A / 2} & (A \in 2 \mathbb{Z}) \\ <\alpha \mid \alpha^{A}=e>=Z_{A} & (A \in 2 \mathbb{Z}+1)\end{cases}
$$

$$
G / D(G) \simeq\left\{\begin{array}{ll}
Z_{2} \times Z_{2}=<\left(d_{\alpha}, d_{\beta}\right) \mid d_{\alpha}^{2}=d_{\beta}^{2}=d_{e}, d_{\alpha} d_{\beta}=d_{\beta} d_{\alpha}=d_{\alpha \beta}> & (A \in 2 \mathbb{Z}) \\
Z_{2}=<d_{\beta} \mid d_{\beta}^{2}=d_{e}> & (A \in 2 \mathbb{Z}+1)
\end{array},\right.
$$

are satisfied, where $\tilde{\alpha} \equiv \alpha^{2}, d_{X} \equiv D(G) X$. Then, in the class (iii), we can obtain

$$
G_{0}=\left\{\begin{array}{ll}
\left\langle\tilde{\alpha}, \tilde{\beta} \mid \tilde{\alpha}^{A / 2}=\tilde{\beta}^{2}=e, \tilde{\beta} \tilde{\alpha} \tilde{\beta}^{-1}=\tilde{\alpha}^{-1}\right\rangle=Z_{A / 2} \rtimes Z_{2} \simeq D_{A / 2} & (A \in 2 \mathbb{Z}) \\
\left\langle\alpha \mid \alpha^{A}=e\right\rangle=Z_{A} & (A \in 2 \mathbb{Z}+1)
\end{array},\right.
$$

$$
G / G_{0} \simeq Z_{N}=Z_{2}=\left\langle g_{\beta} \mid g_{\beta}^{2}=g_{e}\right\rangle, \quad \forall A,
$$

where $\quad \tilde{\beta} \equiv \alpha \beta(x=y=1), \quad g_{\beta} \equiv G_{0} \beta\left(x^{\prime}=0, y^{\prime}=1\right)$. Then, since $\beta^{2}=\tilde{\beta}^{2}=e$, we can write

$$
\begin{aligned}
D_{A}=G & \simeq G_{0} \rtimes Z_{2} \\
& \simeq\left\{\begin{array}{ll}
\left(D(G) \rtimes Z_{2}\right) \rtimes Z_{2} & (A \in 2 \mathbb{Z}) \\
D(G) \rtimes Z_{2} & (A \in 2 \mathbb{Z}+1)
\end{array},\right. \\
& \simeq \begin{cases}D_{A / 2} \rtimes Z_{2} & (A \in 2 \mathbb{Z}) \\
Z_{A} \rtimes Z_{2} & (A \in 2 \mathbb{Z}+1)\end{cases}
\end{aligned}
$$

Here, we comment on the case with $A=2(2 m-1)(m \in$ $\mathbb{Z})$ in particular. In this case, since the coset relation $g_{\beta}=g_{\alpha^{A / 2}}\left(x^{\prime}=A / 2, y^{\prime}=0\right)$ is satisfied and then $k, k^{\prime}, \ell^{\prime}$ are multiples of $A / A^{\prime}=A / 2$, Eq. (44) is satisfied:

$$
\begin{aligned}
D_{A} & =G \simeq D(G) \rtimes\left(Z_{2} \times Z_{2}\right) \simeq Z_{A / 2} \rtimes\left(Z_{2} \times Z_{2}\right), \\
& \simeq G_{0} \times Z_{2} \simeq D_{A / 2} \times Z_{2} .
\end{aligned}
$$

For example, the group $G=S_{3} \simeq D_{3} \simeq A_{3} \rtimes Z_{2}$ is included in this case. Then, we can find that the $S_{3}$ flavor model corresponds to either the class (iii), $G_{0}=D(G)=Z_{3} \simeq A_{3}, G / G_{0} \simeq Z_{N}=Z_{2}$, or the class (i), $G_{0}=G=S_{3}$. It depends on representations including reducible ones. Indeed, $S_{3}$ have three irreducible representations, $\mathbf{1}, \mathbf{1}^{\prime}$, and $\mathbf{2}$, which have $\operatorname{det} \rho_{\mathbf{1}}(\beta)=1, \operatorname{det} \rho_{\mathbf{1}^{\prime}}(\beta)=$ $\operatorname{det} \rho_{\mathbf{2}}(\beta)=-1[2,3]$. The whole $S_{3}$ symmetry is anomaly free in flavor models including even numbers of $\mathbf{1}^{\prime}$ and $\mathbf{2}$. Otherwise, the $Z_{2}$ subsymmetry can be anomalous.

We comment on $G=Q_{A}$ case. $Q_{A}$ group is similar to $D_{A}$ with $A \in 2 \mathbb{Z}$. The difference is $\beta^{2}=\alpha^{A / 2}$ instead of $\beta^{2}=e$. It means that the determinant of the representation of $\beta$ depends on that of $\alpha$, although $D\left(Q_{A}\right)=D\left(D_{A}\right)$. In 
the case of $A \in 4 \mathbb{Z}$, since $\alpha^{A / 2}$ as well as $e$ are included in $D\left(Q_{A}\right)$, the analysis of $Q_{A}$ is same as that of $D_{A}$. However, in the case of $A \in 2(2 \mathbb{Z}+1)$, since $\alpha^{A / 2} \notin D\left(Q_{A}\right), \beta$ becomes anomalous $Z_{4}$ generator in general. This is different from $D_{A}$.

Third, let us consider $G=T_{p^{k}} \simeq Z_{p^{k}} \rtimes Z_{3}$, where $p \neq 3$ and $p \in \mathbb{P}$. If $\operatorname{gcd}\left(m-1, p^{k}\right) \neq 1$, then it should be satisfied that $m=p^{\ell}+1$, where $\ell \in \mathbb{Z} / k \mathbb{Z}-\{0\}$. That requires

$$
\begin{aligned}
& m^{3}-1=p^{\ell}\left(p^{2 \ell}+3 p^{\ell}+3\right) \equiv 0 \quad\left(\bmod p^{k}\right) \\
\Rightarrow & p^{2 \ell}+3 p^{\ell}+3=p^{k-\ell} x, \quad(x \in \mathbb{Z}) \\
& 3=p^{k-\ell} x-p^{2 \ell}-3 p^{\ell}=p^{\ell^{\prime}} y .
\end{aligned}
$$

However, it cannot be satisfied if $p \neq 3$. Thus, we obtain $\operatorname{gcd}\left(m-1, p^{k}\right)=1 \quad$ and then $\quad D(G)=Z_{p^{k}} \subseteq G_{0}$. Therefore, the $T_{p^{k}}$ flavor model corresponds to either the class (iii), $G_{0}=D(G)=Z_{p^{k}}, G / G_{0} \simeq Z_{N}=Z_{3}$, or the class (i), $G_{0}=G=T_{p^{k}}$. In more general, when we discuss $G \simeq Z_{p^{k}} \rtimes Z_{B}$ and $\operatorname{gcd}(p, B)=1$, we obtain $\operatorname{gcd}\left(m-1, p^{k}\right)=1$ and then we find $D(G)=Z_{p^{k}} \subseteq G_{0}$.

Step $2\left(G \simeq\left(Z_{A} \times Z_{A}^{\prime}\right) \rtimes Z_{B}\right) \quad$ Next, let us consider more complicate case, $G \simeq\left(Z_{A} \times Z_{A}^{\prime}\right) \rtimes Z_{B}$. First, let us consider $G=\Sigma\left(2 n^{2}\right) \simeq\left(Z_{n} \times Z_{n}^{\prime}\right) \rtimes Z_{2}$. When we take $\alpha \in Z_{n}, \alpha^{\prime} \in Z_{n}^{\prime}$, and $\beta \in Z_{2}$, they satisfy the following algebraic relations:

$$
\alpha^{n}=\alpha^{\prime n}=\beta^{2}=e,
$$

and also

$$
\alpha^{\prime} \alpha \alpha^{\prime-1}=\alpha \in Z_{n}, \quad\left(\alpha \alpha^{\prime} \alpha^{-1}=\alpha^{\prime} \in Z_{n}^{\prime}\right),
$$

$$
\begin{aligned}
\beta \alpha \beta^{-1} & =\alpha^{m_{1}} \alpha^{\prime m_{2}} \in Z_{n} \times Z_{n}^{\prime}, \\
\beta \alpha^{-1} \beta^{-1} & =\alpha^{m_{3}} \alpha^{\prime m_{4}} \in Z_{n} \times Z_{n}^{\prime} .
\end{aligned}
$$

Here, $m_{i}(i=1,2,3,4)$ can be determined by the constraints, $\beta^{2} \alpha \beta^{-2}=\alpha, \quad \beta^{2} \alpha^{\prime} \beta^{-2}=\alpha^{\prime}$, and then we obtain $m_{1}=m_{4}=0, m_{2}=m_{3}=1$. Thus, Eq. (53) can be rewritten as

$$
\beta \alpha \beta^{-1}=\alpha^{\prime}, \quad \beta \alpha^{\prime} \beta^{-1}=\alpha .
$$

Then, we can find that

$$
D(G)=\left\langle\tilde{\alpha} \mid \tilde{\alpha}^{n}=e\right\rangle=Z_{n},
$$

$G / D(G) \simeq Z_{n} \times Z_{2}=\left\langle\left(d_{\tilde{\alpha}^{\prime}}, d_{\beta}\right)\right| d_{\tilde{\alpha}^{\prime}}^{n}=d_{\beta}^{2}=d_{e}$,

$$
\left.d_{\tilde{\alpha}^{\prime}} d_{\beta}=d_{\beta} d_{\tilde{\alpha}^{\prime}}=d_{\alpha \beta}\right\rangle \text {, }
$$

where $\tilde{\alpha} \equiv \alpha \alpha^{\prime-1}, d_{X} \equiv D(G) X$.

The above situation can also be understood in terms of $Z_{n}$ and $Z_{n}^{\prime}$ charge constraints. Let us assume that the chiral fermions are $Z_{n}$ and $Z_{n}^{\prime}$ eigenstates, where $\left[Z_{n}, Z_{n}^{\prime}\right]$ charges of the $j$ th component field are $\left[q_{j}, q_{j}^{\prime}\right]$. Equation (54) shows that there exists a state with charge $\left[q_{j}^{\prime}, q_{j}\right]$ and the $\beta$ transforms the $j$ th component to the state with charge $\left[q_{j}^{\prime}, q_{j}\right]$. When we consider a fundamental irreducible representation, it becomes a doublet with charge ${ }^{t}\left(\left[q_{1}, q_{1}^{\prime}\right],\left[q_{2}, q_{2}^{\prime}\right]\right)={ }^{t}\left(\left[q, q^{\prime}\right],\left[q^{\prime}, q\right]\right)$, and then we obtain

$$
\operatorname{det} \rho(\alpha)=\operatorname{det} \rho\left(\alpha^{\prime}\right)=e^{2 \pi i\left(q+q^{\prime}\right) / n},
$$

which means Eqs. (55) and (56). Note that there is no constraint for $Z_{2}$ charges.

Similarly, in class (iii), we can obtain

$$
\begin{gathered}
G_{0}=\left\{\begin{array}{ll}
\left\langle\tilde{\alpha}, \tilde{\beta} \mid \tilde{\alpha}^{n}=e, \tilde{\beta}^{2}=\tilde{\alpha}^{n / 2}, \tilde{\beta} \tilde{\alpha} \tilde{\beta}^{-1}=\tilde{\alpha}^{-1}\right\rangle=Q_{n} & (n \in 2 \mathbb{Z}) \\
\left\langle\tilde{\alpha} \mid \tilde{\alpha}^{n}=e\right\rangle=Z_{n} & (n \in 2 \mathbb{Z}+1)
\end{array},\right. \\
G / G_{0} \simeq Z_{N}= \begin{cases}Z_{n}=\left\langle g_{\alpha} \mid g_{\alpha}^{n}=g_{e}\right\rangle \\
Z_{n} \times Z_{2} \simeq Z_{2 n}=\left\langle g_{\tilde{\gamma}} \mid g_{\tilde{\gamma}}^{2 n}=g_{e}\right\rangle & (n \in 2 \mathbb{Z}+1)\end{cases}
\end{gathered}
$$

where $\tilde{\beta} \equiv \alpha^{-n / 2} \beta, \tilde{\gamma} \equiv \alpha^{-(n-1) / 2} \beta, g_{X} \equiv G_{0} X$. We also introduce $\tilde{\alpha}^{\prime} \equiv \tilde{\gamma}^{2}=\tilde{\alpha}^{-(n-1) / 2} \alpha^{\prime}$ and $\tilde{\beta}^{\prime} \equiv \tilde{\gamma}^{n}=\tilde{\alpha}^{-\left(n^{2}-1\right) / 4} \beta$. In particular, since $\alpha^{n}=\tilde{\gamma}^{2 n}\left(=\tilde{\alpha}^{\prime n}=\tilde{\beta}^{\prime 2}\right)=e, G=\Sigma\left(2 n^{2}\right)$ can be written as

$$
\begin{aligned}
\Sigma\left(2 n^{2}\right)=G & \simeq G_{0} \rtimes Z_{N}, \\
& \simeq \begin{cases}Q_{n} \rtimes Z_{n} & (n \in 2 \mathbb{Z}) \\
Z_{n} \rtimes Z_{2 n} & (n \in 2 \mathbb{Z}+1)\end{cases} \\
& \simeq \begin{cases}Q_{n} \rtimes Z_{n} \\
\left(Z_{n} \rtimes Z_{2}\right) \times Z_{n} \simeq D_{n} \times Z_{n} & (n \in 2 \mathbb{Z}+1)\end{cases}
\end{aligned}
$$


Here, the structure of the semidirect product in the case of $n \in 2 \mathbb{Z}$ comes from $\alpha \tilde{\beta} \alpha^{-1}=\tilde{\alpha} \tilde{\beta}$, while the structures of semidirect and direct products in the case of $n \in 2 \mathbb{Z}+1$ come from $\tilde{\beta}^{\prime} \tilde{\alpha} \tilde{\beta}^{\prime-1}=\tilde{\alpha}^{-1}, \tilde{\alpha}^{\prime} \tilde{\alpha} \tilde{\alpha}^{\prime-1}=\tilde{\alpha}, \tilde{\alpha}^{\prime} \tilde{\beta}^{\prime} \tilde{\alpha}^{\prime-1}=\tilde{\beta}^{\prime}$.

This analysis can be easily applied to $G=\Sigma\left(3 n^{3}\right) \simeq$ $\left(Z_{n} \times Z_{n}^{\prime} \times Z_{n}^{\prime \prime}\right) \rtimes Z_{3}$. When we take $\alpha \in Z_{n}, \alpha^{\prime} \in Z_{n}^{\prime}$, $\alpha^{\prime \prime} \in Z_{n}^{\prime \prime}$, and $\beta \in Z_{3}$, they satisfy the following algebraic relations:

$$
\begin{array}{rlrl}
\alpha^{n} & =\alpha^{\prime n}=\alpha^{\prime \prime n}=\beta^{3}=e, \\
\alpha \alpha^{\prime} & =\alpha^{\prime} \alpha, \quad \alpha^{\prime} \alpha^{\prime \prime}=\alpha^{\prime \prime} \alpha^{\prime}, & & \alpha^{\prime \prime} \alpha=\alpha \alpha^{\prime \prime}, \\
\beta \alpha \beta^{-1} & =\alpha^{\prime}, \quad \beta \alpha^{\prime} \beta^{-1}=\alpha^{\prime \prime}, & \beta \alpha^{\prime \prime} \beta^{-1}=\alpha .
\end{array}
$$

Similarly, we can find that

$$
D(G)=\left\langle\tilde{\alpha}, \tilde{\alpha}^{\prime} \mid \tilde{\alpha}^{n}=\tilde{\alpha}^{\prime n}=e, \tilde{\alpha} \tilde{\alpha}^{\prime}=\tilde{\alpha}^{\prime} \tilde{\alpha}\right\rangle=Z_{n} \times Z_{n},
$$

$$
\begin{gathered}
G / D(G) \simeq Z_{n} \times Z_{3}=\left\langle\left(d_{\alpha}, d_{\beta}\right)\right| d_{\alpha}^{n}=d_{\beta}^{3}=d_{e}, \\
\left.d_{\alpha} d_{\beta}=d_{\beta} d_{\alpha}=d_{\alpha \beta}\right\rangle,
\end{gathered}
$$

where $\tilde{\alpha} \equiv \alpha^{\prime} \alpha^{\prime \prime-1}, \tilde{\alpha}^{\prime} \equiv \alpha \alpha^{\prime-1}, d_{X} \equiv D(G) X$. In the class (iii), we can obtain

$$
\begin{gathered}
G_{0}=\left\{\begin{array}{ll}
\left\langle\tilde{\alpha}, \tilde{\alpha}^{\prime}, \tilde{\beta} \mid \tilde{\beta}^{3}=\left(\tilde{\alpha} \tilde{\alpha}^{\prime-1}\right)^{n / 3}, \tilde{\beta} \tilde{\alpha} \tilde{\beta}^{-1}=\tilde{\alpha}^{-1} \tilde{\alpha}^{\prime-1}, \tilde{\beta} \tilde{\alpha}^{\prime} \tilde{\beta}^{-1}=\tilde{\alpha}\right\rangle \equiv R_{n} & (n \in 3 \mathbb{Z}) \\
\left\langle\tilde{\alpha}, \tilde{\alpha}^{\prime}\right\rangle=Z_{n} \times Z_{n} & \text { (otherwise) }
\end{array},\right. \\
G / G_{0} \simeq Z_{N}= \begin{cases}Z_{n}=\left\langle g_{\alpha} \mid g_{\alpha}^{n}=g_{e}\right\rangle & (n \in 3 \mathbb{Z}) \\
Z_{n} \times Z_{3} \simeq Z_{3 n}=\left\langle g_{\tilde{\gamma}} \mid g_{\tilde{\gamma}}^{2 n}=g_{e}\right\rangle & \text { (otherwise) }\end{cases}
\end{gathered}
$$

where $\tilde{\beta} \equiv \alpha^{-n / 3} \beta, \tilde{\gamma} \equiv \alpha^{-(n \mp 1) / 3} \beta, g_{X}=G_{0} X$, and we omit relations between $\tilde{\alpha}$ and $\tilde{\alpha}^{\prime}$ since they are same as Eq. (62). We also introduce $\tilde{\alpha}^{\prime \prime} \equiv \tilde{\gamma}^{3}$ and $\tilde{\beta}^{\prime} \equiv \tilde{\gamma}^{n}$. Here, $R_{n}$ is related to the following $\Delta\left(3 n^{2}\right)$ as with the case that $Q_{n}$ is related to $D_{n}$. Since $\alpha^{n}=\tilde{\gamma}^{3 n}\left(=\tilde{\alpha}^{\prime \prime n}=\tilde{\beta}^{\prime 3}\right)=e$, similarly, $G=\Sigma\left(3 n^{3}\right)$ can be written as

$$
\begin{aligned}
\Sigma\left(3 n^{3}\right)=G & \simeq G_{0} \rtimes Z_{N}, \\
& \simeq\left\{\begin{array}{ll}
R_{n} \rtimes Z_{n} & (n \in 3 \mathbb{Z}) \\
\left(Z_{n} \times Z_{n}\right) \rtimes Z_{3 n} & \text { (otherwise) }
\end{array},\right. \\
& \simeq\left\{\begin{array}{ll}
R_{n} \rtimes Z_{n} & (n \in 3 \mathbb{Z}) \\
\left(\left(Z_{n} \times Z_{n}\right) \rtimes Z_{3}\right) \times Z_{n} \simeq \Delta\left(3 n^{2}\right) \times Z_{n} & \text { (otherwise) }
\end{array} .\right.
\end{aligned}
$$

Here, the structure of the semidirect product in the case of $n \in 3 \mathbb{Z}$ comes from $\alpha \tilde{\beta} \alpha^{-1}=\tilde{\alpha}^{\prime} \tilde{\beta}$, while the structures of semidirect and direct products in the case of $n \in 3 \mathbb{Z} \pm 1$ come from $\tilde{\beta}^{\prime} \tilde{\alpha} \tilde{\beta}^{\prime-1}=\tilde{\alpha}^{-1} \tilde{\alpha}^{\prime-1}, \tilde{\beta}^{\prime} \tilde{\alpha}^{\prime} \tilde{\beta}^{\prime-1}=\tilde{\alpha}, \tilde{\alpha}^{\prime \prime} \delta \tilde{\alpha}^{\prime \prime-1}=$ $\delta\left(\delta=\tilde{\alpha}, \tilde{\alpha}^{\prime}, \tilde{\beta}^{\prime}\right)$.

Next, let us see another example, $G=\Delta\left(3 n^{2}\right) \simeq$ $\left(Z_{n} \times Z_{n}^{\prime}\right) \rtimes Z_{3}$. When we take $\alpha \in Z_{n}, \alpha^{\prime} \in Z_{n}^{\prime}$, and $\beta \in Z_{3}$, they satisfy the following algebraic relations:

$$
\alpha^{n}=\alpha^{\prime n}=\beta^{3}=e,
$$

and also

$$
\alpha^{\prime} \alpha \alpha^{\prime-1}=\alpha \in Z_{n}, \quad\left(\alpha \alpha^{\prime} \alpha^{-1}=\alpha^{\prime} \in Z_{n}^{\prime}\right),
$$

$$
\begin{aligned}
\beta \alpha \beta^{-1} & =\alpha^{m_{1}} \alpha^{\prime m_{2}} \in Z_{n} \times Z_{n}^{\prime}, \\
\beta \alpha^{-1} \beta^{-1} & =\alpha^{m_{3}} \alpha^{\prime m_{4}} \in Z_{n} \times Z_{n}^{\prime} .
\end{aligned}
$$

Here, $m_{i}(i=1,2,3,4)$ can be determined by the constraints, $\beta^{3} \alpha \beta^{-3}=\alpha, \beta^{3} \alpha^{\prime} \beta^{-3}=\alpha^{\prime}$, and then we obtain $m_{1}=m_{2}=-1, m_{3}=1, m_{4}=0$. Thus, Eq. (69) can be rewritten as

$$
\beta \alpha \beta^{-1}=\alpha^{-1} \alpha^{\prime-1}, \quad \beta \alpha^{\prime} \beta^{-1}=\alpha .
$$

Then we can obtain

$$
D(G)= \begin{cases}\left\langle\tilde{\alpha}, \tilde{\alpha}^{\prime} \mid \tilde{\alpha}^{n}=\tilde{\alpha}^{\prime n / 3}=e, \tilde{\alpha} \tilde{\alpha}^{\prime}=\tilde{\alpha}^{\prime} \tilde{\alpha}\right\rangle=Z_{n} \times Z_{n / 3} & (n \in 3 \mathbb{Z}) \\ \left\langle\tilde{\alpha}, \tilde{\alpha}^{\prime} \mid \tilde{\alpha}^{n}=\tilde{\alpha}^{\prime n}=e, \tilde{\alpha} \tilde{\alpha}^{\prime}=\tilde{\alpha}^{\prime} \tilde{\alpha}\right\rangle=Z_{n} \times Z_{n} & (\text { otherwise })\end{cases}
$$




$$
G / D(G) \simeq \begin{cases}Z_{3} \times Z_{3}=\left\langle\left(d_{\alpha}, d_{\beta}\right) \mid d_{\alpha}^{3}=d_{\beta}^{3}=d_{e}, d_{\alpha} d_{\beta}=d_{\beta} d_{\alpha}=d_{\alpha \beta}\right\rangle & (n \in 3 \mathbb{Z}) \\ Z_{3}=\left\langle d_{\beta} \mid d_{\beta}^{3}=d_{e}\right\rangle & \text { (otherwise) }\end{cases}
$$

where $\tilde{\alpha} \equiv \alpha \alpha^{\prime-1}, \tilde{\alpha}^{\prime} \equiv \alpha^{-3}, d_{X} \equiv D(G) X$.

The above situation can also be understood in terms of $Z_{n}$ and $Z_{n}^{\prime}$ charge constraints. Let us assume that the chiral fermions are $Z_{n}$ and $Z_{n}^{\prime}$ eigenstates, where the $\left[Z_{n}, Z_{n}^{\prime}\right]$ charges of the $j$ th component field are $\left[q_{j}, q_{j}^{\prime}\right]$. Equation (70) shows that there exists a state with charge $\left[-\left(q_{j}+q_{j}^{\prime}\right), q_{j}\right]$ and $\beta$ transforms the $j$ th component to the sate with charge $\left[-\left(q_{j}+q_{j}^{\prime}\right), q_{j}\right]$. When we consider a fundamental irreducible representation, it becomes a triplet with charge ${ }^{t}\left(\left[q_{1}, q_{1}^{\prime}\right],\left[q_{2}, q_{2}^{\prime}\right],\left[q_{3}, q_{3}^{\prime}\right]\right)={ }^{t}\left(\left[q, q^{\prime}\right]\right.$, $\left.\left[-\left(q+q^{\prime}\right), q\right],\left[q^{\prime},-\left(q+q^{\prime}\right)\right]\right)$, and then we have

$$
\operatorname{det} \rho(\alpha)=\operatorname{det} \rho\left(\alpha^{\prime}\right)=1
$$

which means Eqs. (71) and (72). Note that for the triplet, Eq. (73) and also det $\rho(\beta)=1$ are satisfied, even if $n \in 3 \mathbb{Z}$. In the class (iii), we can obtain

$$
G_{0}= \begin{cases}\left\langle\tilde{\alpha}, \tilde{\alpha}^{\prime}, \tilde{\beta} \mid \tilde{\beta}^{3}=e, \tilde{\beta} \tilde{\alpha} \tilde{\beta}^{-1}=\tilde{\alpha} \tilde{\alpha}^{\prime}, \tilde{\beta} \tilde{\alpha}^{\prime} \tilde{\beta}^{-1}=\tilde{\alpha}^{-3} \tilde{\alpha}^{\prime-2}\right\rangle=\left(Z_{n} \times Z_{n / 3}\right) \rtimes Z_{3} & (n \in 3 \mathbb{Z}) \\ \left\langle\tilde{\alpha}, \tilde{\alpha}^{\prime}\right\rangle=Z_{n} \times Z_{n} & \text { (otherwise) }\end{cases}
$$

$$
G / G_{0} \simeq Z_{N}=Z_{3}=\left\langle g_{\beta} \mid g_{\beta}^{3}=g_{e}\right\rangle, \quad \forall n,
$$

where $\tilde{\beta} \equiv \alpha^{-1} \beta, g_{\beta} \equiv G_{0} \beta$, and we omit relations between $\tilde{\alpha}$ and $\tilde{\alpha}^{\prime}$ since they are same as Eq. (71). Then, since $\beta^{3}=\tilde{\beta}^{3}=e$, we can write

$$
\begin{aligned}
\Delta\left(3 n^{2}\right)=G & \simeq G_{0} \rtimes Z_{3}, \\
& \simeq\left\{\begin{array}{ll}
\left(D\left(\Delta\left(3 n^{2}\right)\right) \rtimes Z_{3}\right) \rtimes Z_{3} & (n \in 3 \mathbb{Z}) \\
D\left(\Delta\left(3 n^{2}\right)\right) \rtimes Z_{3} & \text { (otherwise) }
\end{array},\right. \\
& \simeq \begin{cases}\left(\left(Z_{n} \times Z_{n / 3}\right) \rtimes Z_{3}\right) \rtimes Z_{3} & (n \in 3 \mathbb{Z}) \\
\left(Z_{n} \times Z_{n}\right) \rtimes Z_{3} & \text { (otherwise) })\end{cases}
\end{aligned}
$$

where the last $Z_{3}$ semidirect product in the case of $n \in 3 \mathbb{Z}$ comes from $\beta \tilde{\alpha} \beta^{-1}=\tilde{\alpha} \tilde{\alpha}^{\prime}, \quad \beta \tilde{\alpha}^{\prime} \beta^{-1}=\tilde{\alpha}^{-3} \tilde{\alpha}^{\prime-2}, \beta \tilde{\beta} \beta^{-1}=$ $\tilde{\alpha}^{-1} \tilde{\alpha}^{\prime-1} \tilde{\beta}$. For example, the group $G=A_{4} \simeq \Delta(12)$ is included in this case, and then we can find that the $A_{4}$ flavor model corresponds to either the class (iii), $G_{0}=D(G)=Z_{n} \times Z_{n}^{\prime}, G / G_{0} \simeq Z_{N}=Z_{3}$, or the class (i), $G_{0}=G=A_{4}$. Indeed, the $A_{4}$ symmetry has four irreducible representations, $\mathbf{1}, \mathbf{1}^{\prime}, \mathbf{1}^{\prime \prime}$, and $\mathbf{3}$, which have $\operatorname{det} \rho_{\mathbf{1}}(\beta)=\operatorname{det} \rho_{\mathbf{3}}(\beta)=1, \operatorname{det} \rho_{\mathbf{1}^{\prime}}(\beta)=e^{2 \pi i / 3}$ and $\operatorname{det} \rho_{\mathbf{1}^{\prime \prime}}(\beta)=e^{4 \pi i / 3} \quad[2,3]$. The whole $A_{4}$ symmetry is anomaly free in flavor models including a proper number of $\mathbf{1}^{\prime}$ and $\mathbf{1}^{\prime \prime}$. Otherwise, the $Z_{3}$ subsymmetry can be anomalous. We note that, in the case of double covering group $T^{\prime}$, there is no modification except double covering.

Step $3\left(G \simeq K_{G} \rtimes Z_{B}\right)$ The above two types in the steps 1 and 2 are specific examples of $G \simeq K_{G} \rtimes Z_{B}$ type. Then, let us consider a more generic case, $G \simeq K_{G} \rtimes Z_{B}\left(G / K_{G} \simeq Z_{B}\right)$. In this case, it is found that $D(G) \subseteq K_{G}$. Then, we can find that

$$
G / D(G) \simeq\left(K_{G} / D(G)\right) \times Z_{B}
$$

from the following proof.

Proof.-We can prove Eq. (77)

(I) From the isomorphism Theorem 3 in Appendix A, it is satisfied that

$$
Z_{B} \simeq G / K_{G} \simeq(G / D(G)) /\left(K_{G} / D(G)\right) .
$$

(II) Since $G \simeq K_{G} \rtimes Z_{B}, Z_{B}$ is a subgroup of $G$ and the relation $K_{G} \cap Z_{B}=\{e\}$ is satisfied. Then, the relation $\left(K_{G} / D(G)\right) \cap Z_{B}=\{e\}$ is also satisfied. Thus, we can write $G / D(G) \simeq\left(K_{G} / D(G)\right) \rtimes Z_{B}$.

(III) In particular, since $G / D(G)$ is Abelian, $G / D(G) \simeq\left(K_{G} / D(G)\right) \times Z_{B}$.

Then, in the class (iii), $G / G_{0} \simeq Z_{N}$ can be obtained by calculating the least common multiple of all orders of cyclic groups.

As an example which is not mentioned in the steps 1 and 2 , let us consider $G=S_{n} \simeq A_{n} \rtimes Z_{2}(n \geq 5)$. Since $A_{n}(n \geq 5)$ is a perfect group, $A_{n}$ should be included in $D(G), A_{n} \subseteq D(G)$, and then we find $D(G)=A_{n}$. Thus, we can find that the $S_{n}$ flavor model corresponds to either the class (iii), $G_{0}=D(G)=A_{n}, G / G_{0} \simeq Z_{N}=Z_{2}$, or the class (i), $G_{0}=G=S_{n}$.

Step 4 (General $G \simeq K_{G} \rtimes G^{(1)}$ ) Finally, let us consider general case $G \simeq K_{G} \rtimes G^{(1)}\left(G / K_{G} \simeq G^{(1)}\right)$. When $G^{(1)}$ is Abelian, it comes down to the step 3 because of the fundamental structure theorem of finite Abelian group in Appendix C and Eqs. (B4) and (B5). Generally, as discussed in the step 3 , we can obtain 


$$
G / D(G) \simeq\left(K_{G} / D(G)\right) \times G^{(1)} \quad(\text { Abelian }) .
$$

Now, let us consider the case that $G^{(1)}$ is non-Abelian. Since $G / K_{G} \simeq G^{(1)}$ and $G^{(1)} \triangleright D\left(G^{(1)}\right) \neq\{e\}$, by use of the correspondence theorem in Appendix A, the relation $K_{G} \subset D(G) \subseteq G_{0}$ is satisfied. Here, similar to $G_{0}$, we define $G_{0}^{(1)} \equiv\left\{g_{0}^{(1)} \in G^{(1)} \mid \operatorname{det} \rho\left(g_{0}^{(1)}\right)=1\right\}$, and then we see $D\left(G^{(1)}\right) \subseteq G_{0}^{(1)}$. By use of the isomorphism theorem 3 in Appendix A, we can find that

$$
\begin{aligned}
Z_{N} & \simeq G / G_{0}, \\
& \simeq(G / D(G)) /\left(G_{0} / D(G)\right), \\
& \simeq\left[\left(G / K_{G}\right) /\left(D(G) / K_{G}\right)\right] /\left[\left(G_{0} / K_{G}\right) /\left(D(G) / K_{G}\right)\right], \\
& \simeq\left(G^{(1)} / D\left(G^{(1)}\right)\right) /\left(G_{0}^{(1)} / D\left(G^{(1)}\right)\right), \\
& \simeq G^{(1)} / G_{0}^{(1)} .
\end{aligned}
$$

Therefore, in this case, the structures of $D(G), G / D(G)$, $G_{0}$, and $G / G_{0}$ depend on $D\left(G^{(1)}\right), G^{(1)} / D\left(G^{(1)}\right), G_{0}^{(1)}$, and $G^{(1)} / G_{0}^{(1)}$, respectively.

For example, let us consider $G=\Delta\left(6 n^{2}\right)$,

$$
\begin{aligned}
\Delta\left(6 n^{2}\right) & \simeq\left(Z_{n} \times Z_{n}^{\prime}\right) \rtimes S_{3} \simeq\left(Z_{n} \times Z_{n}^{\prime}\right) \rtimes\left(Z_{3} \rtimes Z_{2}\right), \\
& \simeq\left(\left(Z_{n} \times Z_{n}^{\prime}\right) \rtimes Z_{3}\right) \rtimes Z_{2} \simeq \Delta\left(3 n^{2}\right) \rtimes Z_{2} .
\end{aligned}
$$

Then, we can find that $Z_{n} \times Z_{n}^{\prime} \subset G_{0}$ and $Z_{N} \simeq S_{3} / G_{0}^{(1)}$. In addition, it is satisfied that $S_{3} \simeq A_{3} \rtimes Z_{2} \simeq Z_{3} \rtimes Z_{2}$ and
$G_{0}^{(1)} \supseteq A_{3} \simeq Z_{3}$. Thus, we can find that $\Delta\left(6 n^{2}\right)$ flavor model corresponds to either the class (iii), $G_{0}=D(G)=\Delta\left(3 n^{2}\right), \quad G / G_{0} \simeq Z_{N}=Z_{2}$, or the class (i), $G_{0}=G=\Delta\left(6 n^{2}\right)$. For example, $\quad S_{4} \simeq \Delta(24) \simeq$ $\Delta(12) \rtimes Z_{2} \simeq A_{4} \rtimes Z_{2}$ is included in this case. Indeed, the $S_{4}$ symmetry has five irreducible representations, $\mathbf{1}, \mathbf{1}^{\prime}$, $\mathbf{2}, \mathbf{3}$, and $\mathbf{3}^{\prime}$, which have $\operatorname{det} \rho_{\mathbf{1}}(\beta)=\operatorname{det} \rho_{\mathbf{3}^{\prime}}(\beta)=1$, $\operatorname{det} \rho_{\mathbf{1}^{\prime}}(\beta)=\operatorname{det} \rho_{\mathbf{2}}(\beta)=\operatorname{det} \rho_{\mathbf{3}}(\beta)=-1[2,3]$. The whole $S_{4}$ symmetry is anomaly free in flavor models including even numbers of $\mathbf{1}^{\prime}, \mathbf{2}$, and $\mathbf{3}$. Otherwise, the $Z_{2}$ subsymmetry can be anomalous.

So far, we have seen the detail structure of the anomalyfree subgroup $G_{0}$ and the anomalous part $G / G_{0} \simeq Z_{N}$ for various typical groups $G$ from the structure of the derived subgroup $D(G)$ and the residue class group $G / D(G)$. Here, we list $D(G)$ of those typical groups $G$ and $G / D(G)$. We note again that the derived subgroup $D(G)$ is also automatically the subgroup of anomaly-free group $G_{0}$, which holds in any arbitrary representations. From the following Table I, when we consider $G=S_{n} \simeq A_{n} \rtimes Z_{2}$ and $G=\Delta\left(6 n^{2}\right) \simeq \Delta\left(3 n^{2}\right) \rtimes Z_{2}$, in particular, we can find that $G_{0} \supseteq A_{n}$ and $G_{0} \supseteq \Delta\left(3 n^{2}\right)$ are at least satisfied, respectively.

Finally, let us summarize the important points in this section again.

(i) The derived subgroup of $G, D(G)$, in Eq. (12) is always included in $G_{0}$, i.e., $D(G) \subseteq G_{0}$ $\left(D(G) \triangleleft G_{0}\right)$. It does not depend on representations of $G$. While $G / G_{0} \simeq Z_{N}, G / D(G)$ becomes Abelian

TABLE I. The derived subgroup $D(G)$ of typical groups $G$ and the residue class group $G / D(G)$.

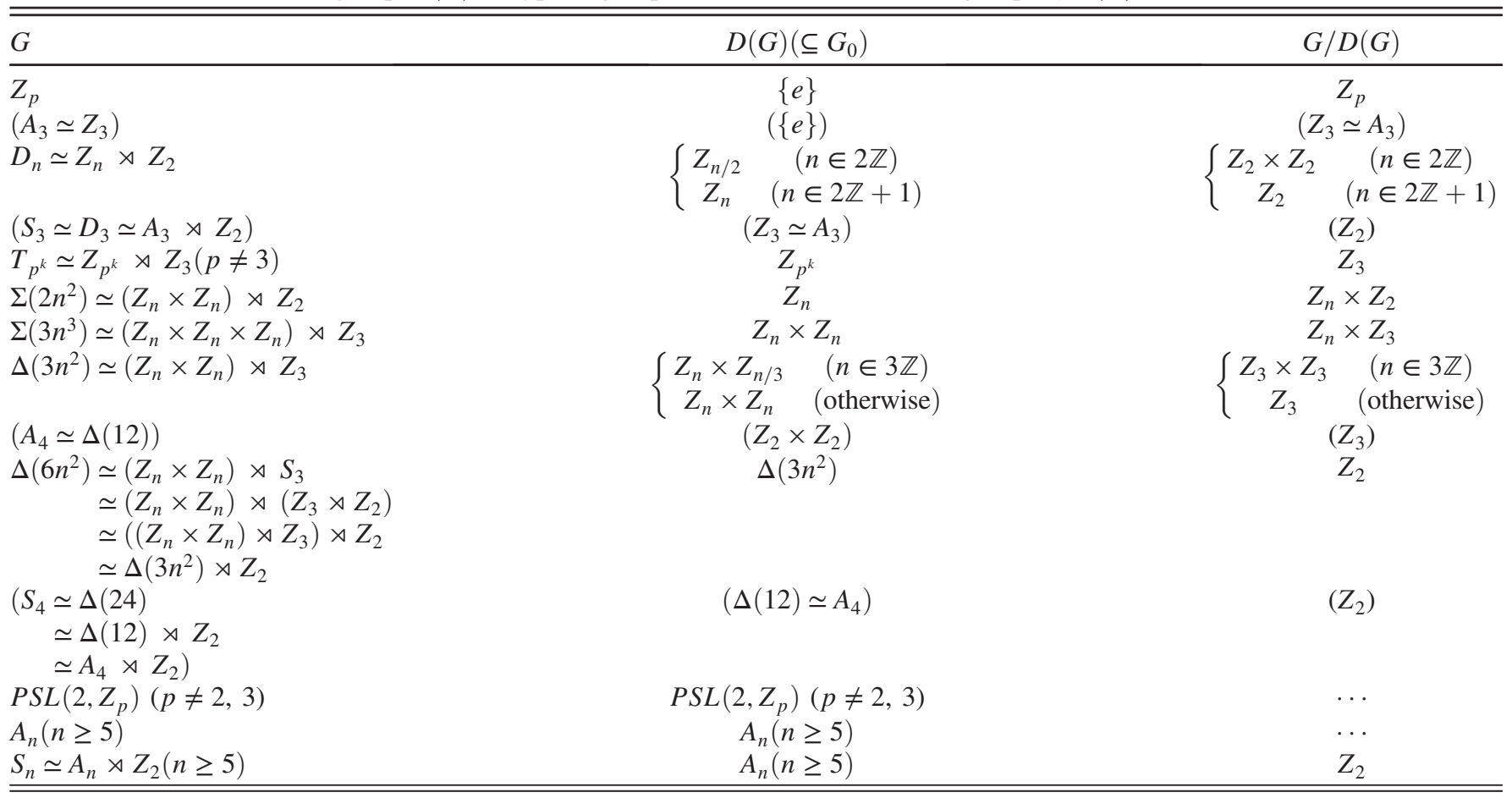


in Eq. (15) and each cyclic groups can be anomalous. The number $N$ can be found by the least common multiple of orders of the anomalous cyclic subgroups. Thus, $D(G)$ gives an important clue to obtain information about the anomaly-free subgroup $G_{0}$ and the anomalous part $G / G_{0} \simeq Z_{N}$.

(ii) The detail structure of $D(G)$ depends on the structure of $G$. However, if $G$ can be written as $G \simeq K_{G} \rtimes G^{(1)}$, we can obtain some information as discussed in the above step 4 ; in the case that $G^{(1)}$ is Abelian, the relations $D(G) \subseteq K_{G}$ and $G / D(G) \simeq$ $\left(K_{G} / D(G)\right) \times G^{(1)}$ are satisfied, while in the case that $G^{(1)}$ is non-Abelian, the relations $K_{G} \subset D(G) \subseteq$ $G_{0}$ and $G / D(G) \simeq G^{(1)} / D\left(G^{(1)}\right)$ are satisfied. In particular, when we consider $G=S_{n} \simeq A_{n} \rtimes Z_{2}$ and $G=\Delta\left(6 n^{2}\right) \simeq \Delta\left(3 n^{2}\right) \rtimes Z_{2}$, we find $G_{0} \supseteq$ $A_{n}$ and $G_{0} \supseteq \Delta\left(3 n^{2}\right)$ at least.

\section{COMMENT ON GENERIC THEORIES WITH $M>1$}

We have concentrated on the theory with $\sum_{\mathbf{R}} 2 T_{2}(\mathbf{R})=1$ and the anomaly-free condition $\operatorname{det} \rho(g)=1$. In this theory, the anomaly-free elements correspond to a normal subgroup $G_{0}$ of $G$. Then, the residue class group $G / G_{0} \simeq Z_{N}$ can be anomalous. We can extend our analysis to the theory with $\sum_{\mathbf{R}} 2 T_{2}(\mathbf{R})=M>1$ and anomaly-free condition $(\operatorname{det} \rho(g))^{M}=1$.

When the determinant of the representation of any element $\forall g \in G$ satisfies $(\operatorname{det} \rho(g))^{N}=1$, that of the anomaly-free element $g_{n}$ satisfies $\left(\operatorname{det} \rho\left(g_{n}\right)\right)^{n}=1$ with $n=\operatorname{gcd}(N, M)$, which means the determinant can be written as $\operatorname{det} \rho\left(g_{n}\right)=e^{2 \pi i Q^{\prime \prime}\left(g_{n}\right) / n}$. Then, we define the subset of $G$,

$G_{n} \equiv\left\{g_{n} \in G \mid \operatorname{det} \rho\left(g_{n}\right)=e^{2 \pi i Q^{\prime}\left(g_{n}\right) / N}=e^{2 \pi i Q^{\prime \prime}\left(g_{n}\right) / n}\right\}$,

where $Q^{\prime}\left(g_{n}\right)\left(=Q\left(g_{n}\right) N / N\left(g_{n}\right)\right)=Q^{\prime \prime}\left(g_{n}\right) N / n$. Similar to $G_{0}$, we find that $G_{n}$ is also a normal subgroup of $G$, $G_{n} \triangleleft G$. In addition, $G_{n}$ includes of $G_{0}, G_{0} \subset G_{n}$. That also means $G_{0} \triangleleft G_{n}$. Then, we can similarly derive $G / G_{n} \simeq Z_{N / n}$ and $G_{n} / G_{0} \simeq Z_{n}$. Indeed, by use of the isomorphism Theorem 3 in Appendix A, we find that

$$
G / G_{n} \simeq\left(G / G_{0}\right) /\left(G_{n} / G_{0}\right) \simeq Z_{N} / Z_{n} \simeq Z_{N / n} .
$$

That is, in this particle theory, the subgroup $G_{n}$ is always anomaly free, but $Z_{N / n}$ can be anomalous. Thus, the anomalous symmetry is the single cyclic group again. Furthermore, if there exists $\exists g \in G$ which satisfies $N(g)=N / n$ and $\operatorname{gcd}(Q(g), N(g))=1$, then $G$ can be expressed as

$$
G \simeq G_{n} \rtimes Z_{N / n} .
$$

In this case, the anomaly-free and anomalous parts of $G$ can be separated.

There is an interesting example. When $M$ is a multiple of $N$, the whole symmetry $G$ is anomaly free. As the example, let us assume that our particle theory has $E_{6}$ gauge symmetry $^{6}$ and all chiral fermions in the theory transform as $\mathbf{2 7}^{i}$ representation under $E_{6}$ transformation, where $i$ denotes the flavor index. (For example, the $i$ th generational standard model quarks and leptons are embedded in $27^{i}$ representation.) Furthermore, we also assume non-Abelian discrete flavor symmetry $G$ among those chiral fermions at least at classical level. In this case, we find that $M=2 T_{2}(\mathbf{2 7})=6$. Then, from Eq. (26), when $G$ corresponds to either of the groups listed in Table I except $\Sigma\left(2 n^{2}\right)$ and $\Sigma\left(3 n^{2}\right)$, at least, we can find that whole $G$ flavor symmetry can be always anomaly free whatever the fermions have any representations of $G$.

\section{CONCLUSION}

We have studied the anomaly-free subgroup $G_{0}$ of a discrete group $G$. If the determinant of a chiral transformation is trivial, det $\rho\left(g_{0}\right)=1\left(g_{0} \in G\right)$, in particular, then the transformation is anomaly free. We have found that the anomaly-free transformations generate a normal subgroup of the group $G, G_{0} \triangleleft G$, and the residue class group $G / G_{0}$, which becomes the anomalous part of $G$, which is isomorphic to a single cyclic group $Z_{N}$, where the number $N$ can be read from $\operatorname{det} \rho(g)=e^{2 \pi i Q^{\prime}(g) / N}(\forall g \in G)$. In particular, if this $Z_{N}$ actually becomes the subgroup of $G, G$ can be written as $G \simeq G_{0} \rtimes Z_{N}$, which means that the anomaly-free and anomalous parts can be separated. Furthermore, this structure constrains the structure of $G_{0} ; G_{0}$ has to contain the derived subgroup $D(G)$ in Eq. (12) due to $G / G_{0} \simeq Z_{N}$, and $D(G)$ is also a normal subgroup of $G_{0}$. In addition, the number $N$ is also constrained as a divisor of the least common multiple of orders of each cyclic group of $G / D(G)$. In this sense, $D(G)$ and $G / D(G)$ give an important clue to obtain information about the anomaly-free subgroup $G_{0}$ and the anomalous part $G / G_{0} \simeq Z_{N}$.

Then, we have studied their detail structure in various discrete groups $G$. In particular, if $G$ can be written as $G \simeq K_{G} \rtimes G^{(1)}$, we can obtain some information; in the case that $G^{(1)}$ is Abelian, we find $D(G) \subseteq K_{G}$ and $G / D(G) \simeq\left(K_{G} / D(G)\right) \times G^{(1)}$, while in the case that $G^{(1)}$ is non-Abelian, we find $K_{G} \subset D(G) \subseteq G_{0}$ and $G / D(G) \simeq G^{(1)} / D\left(G^{(1)}\right)$. Interestingly, when we consider $G=S_{n} \simeq A_{n} \rtimes Z_{2}$ and $G=\Delta\left(6 n^{2}\right) \simeq \Delta\left(3 n^{2}\right) \rtimes Z_{2}$, we obtain $G_{0} \supseteq A_{n}$ and $G_{0} \supseteq \Delta\left(3 n^{2}\right)$ at least. This result holds in any arbitrary representations. We would like to study some applications elsewhere.

\footnotetext{
${ }^{6} E_{6}$ gauge symmetry is automatically anomaly free.
} 
These analyses can be extended to particle theories with $\sum_{\mathbf{R}} 2 T_{2}(\mathbf{R})=M>1$. Then, the group $G$ has the anomaly-free normal subgroup $G_{n}$ and then the residue class group $G / G_{n} \simeq Z_{N / n}$ becomes the anomalous part, where $n=\operatorname{gcd}(N, M)$. Similarly, if this $Z_{N / n}$ actually becomes the subgroup of $G, G$ can be written as $G \simeq G_{n} \rtimes Z_{N / n}$. Interestingly, if we consider that the particle theory has $E_{6}$ gauge symmetry and a discrete flavor symmetry $G$, and all chiral fermions in the theory are represented as $\mathbf{2 7}$ under $E_{6}$, at least most of groups $G$ in Table I can be always anomaly free whatever the fermions have any representations of $G$.

We comment the Green-Schwarz mechanism for anomaly cancellation in four-dimensional low-energy effective field theory derived from superstring theory. (See for review Refs. [16,17].) Some of $U(1)$ gauge symmetries are anomalous in such effective field theory, but those anomalies can be canceled by the Green-Schwarz mechanism, where axions shift under the anomalous $U(1)$ symmetries. If the cyclic symmetry $G / G_{0} \simeq Z_{N}$ can be embedded into those anomalous $U(1)$ symmetries, anomalies of the $Z_{N}$ symmetry can also be canceled by the same Green-Schwarz mechanism. ${ }^{7}$ From this point, it would be important that only the single $Z_{N}$ symmetry is anomalous but not generic Abelian group.

\section{ACKNOWLEDGMENTS}

Authors would like to thank S. Kikuchi, K. Nasu, and S. Takada for useful discussions. H. U. was supported by Grant-in-Aid for JSPS Research Fellows No. JP20J20388.

\section{APPENDIX A: ISOMORPHISM THEOREMS}

We introduce the fundamental homomorphism and then the isomorphism theorems as well as the correspondence theorem.

Fundamental homomorphism theorem Let $K$ be a normal subgroup of $G, K \triangleleft G$, and then there is a natural homomorphism $\pi: G \rightarrow G / K$. Let $f: G \rightarrow G^{\prime}$ be a group homomorphism. In this case, $\operatorname{Ker}(f) \triangleleft G$ and we can consider $G^{\prime}=\operatorname{Im}(f)$ without loss of generality. If $K$ is a subset of $\operatorname{Ker}(f), K \subseteq \operatorname{Ker}(f)$, then there exists a unique homomorphism $F: G / K \rightarrow G^{\prime}$ such that $F \circ \pi=f$.

Isomorphism theorem In particular, if $K=\operatorname{Ker}(f)$, $F: G / K \rightarrow G^{\prime}(=\operatorname{Im}(f))$ becomes a isomorphism, then

$$
G / \operatorname{Ker}(f) \simeq \operatorname{Im}(f) .
$$

This is always satisfied when we consider $f: G \rightarrow G^{\prime}$.

Correspondence theorem Then, it can be applied even if $K \subset \operatorname{Ker}(f)$. In this case, $G / K \triangleright \operatorname{Ker}(F) \neq\{e\}$ and then

\footnotetext{
${ }^{7}$ See for examples Refs. [13,18,19].
}

$$
(G / K) / \operatorname{Ker}(F) \simeq G^{\prime} \simeq G / \operatorname{Ker}(f)
$$

is satisfied. Here, $\quad K \subset \operatorname{Ker}(f)=\operatorname{Ker}(F \circ \pi)=$ $\pi^{-1}(\operatorname{Ker}(F)) \triangleleft G$. Furthermore, there exists a group homomorphism $\phi: G \rightarrow G^{\prime \prime}$, which satisfies $\operatorname{Ker}(\phi)=K$ and then $G^{\prime \prime} \simeq G / K$. Accordingly, there exists $\tilde{K}^{\prime \prime} \triangleleft G^{\prime \prime}$ such that $\tilde{K}^{\prime \prime} \simeq \operatorname{Ker}(F)$. Thus, for $\phi: G \rightarrow G^{\prime \prime}$,

$$
G / \tilde{K} \simeq G^{\prime \prime} / \tilde{K}^{\prime \prime}
$$

is satisfied in general, where $\tilde{K}^{\prime \prime} \triangleleft G^{\prime \prime}$ and $\tilde{K} \equiv$ $\phi^{-1}\left(\tilde{K}^{\prime \prime}\right)(\supset \operatorname{Ker}(\phi)) \triangleleft G$. This is often called the corresponding theorem.

Isomorphism theorem Let $K$ be a normal subgroup of $G, K \triangleleft G$. Let $H$ be a subgroup of $G, H \subset G$. In this case, $K$ is also a normal subgroup of $K H$. In particular, $H \cap K$ is also a normal subgroup of $H$. When we consider $\phi: K H \rightarrow H, \phi(K)=H \cap K$ and then

$$
K H / K \simeq H /(H \cap K)
$$

is satisfied.

Isomorphism theorem Let both $K_{1}$ and $K_{2}$ be normal subgroups of $G$ which satisfy $K_{1} \subset K_{2}$. In this case, $K_{1} \triangleleft$ $K_{2}$ and $K_{2} / K_{1} \triangleleft G / K_{1}$. By considering the corresponding theorem,

$$
G / K_{2} \simeq\left(G / K_{1}\right) /\left(K_{2} / K_{1}\right)
$$

is satisfied.

\section{APPENDIX B: SEMIDIRECT PRODUCT}

We comment on semidirect product. If a normal subgroup of $G, K_{G}$, and a subgroup of $G, G^{(1)}$ satisfy the following conditions,

$$
G=K_{G} G^{(1)}, \quad K_{G} \cap G^{(1)}=\{e\},
$$

then $G$ can be expressed as

$$
G \simeq K_{G} \rtimes G^{(1)} .
$$

In particular, if any elements of $G^{(1)}$ commute all elements of $K_{G}$, then $G$ can be expressed as $G \simeq K_{G} \times G^{(1)}$. Applying the second isomorphism theorem in Appendix A to this, we can find $G / K_{G} \simeq G^{(1)}$. We note that $G$ cannot be always expressed as $G \simeq K_{G} \rtimes G^{(1)}$ just because $G / K_{G} \simeq G^{(1)}$. If this $G^{(1)}$ is actually a subgroup of $G$, then Eq. (B1) is satisfied and then $G$ can be expressed as Eq. (B2). In terms of components of Eq. (B2), since $K_{G}$ is the normal subgroup of $G$, 


$$
g_{1} k g_{1}^{-1}=k^{\left(g_{1}\right)} \in K_{G},
$$

should be satisfied, where $k, k^{\left(g_{1}\right)} \in K_{G}$ and $g_{1} \in G^{(1)}$.

Now, let us see the case that $G^{(1)}$ is further expressed as $G^{(1)} \simeq K_{G^{(1)}} \rtimes G^{(2)}$. In this case, $G$ can be expressed as

$$
\begin{aligned}
& \simeq \simeq K_{G} \rtimes G^{(1)} \simeq K_{G} \rtimes\left(K_{G^{(1)}} \rtimes G^{(2)}\right) \\
\Rightarrow & G \simeq\left(K_{G} \rtimes K_{G^{(1)}}\right) \rtimes G^{(2)} \simeq K_{G}^{\prime} \rtimes G^{(2)} .
\end{aligned}
$$

This can be found as the following by considering relations of their elements, $k, k^{\left(k_{1}\right)}, k^{\left(g_{2}\right)}, k^{\left(g_{2} \rightarrow k_{1}\right)} \in K_{G}, k_{1}, k_{1}^{\left(g_{2}\right)}$, $k_{1}^{\left(g_{2} \rightarrow k\right)} \in K_{G^{(1)}}$, and $g_{2} \in G^{(2)}$. In the case of Eq. (B4),

$k_{1} k k_{1}^{-1}=k^{\left(k_{1}\right)}, \quad g_{2} k g_{2}^{-1}=k^{\left(g_{2}\right)}, \quad g_{2} k_{1} g_{2}^{-1}=k_{1}^{\left(g_{2}\right)}$

are satisfied, while in the case of Eq. (B5),

$$
\begin{aligned}
k_{1} k k_{1}^{-1} & =k^{\left(k_{1}\right)}, \quad g_{2} k g_{2}^{-1}=k^{\left(g_{2}\right)} k_{1}^{\left(g_{2} \rightarrow k\right)}, \\
g_{2} k_{1} g_{2}^{-1} & =k^{\left(g_{2} \rightarrow k_{1}\right)} k_{1}^{\left(g_{2}\right)}
\end{aligned}
$$

are satisfied. Thus, Eq. (B6) is sufficient condition for Eq. (B7), but Eq. (B7) does not always satisfy Eq. (B6).

\section{APPENDIX C: FINITED ABELIAN GROUPS}

We introduce some theorems of finite Abelian groups.
Fundamental structure theorem of finite Abelian group Every finite Abelian group $G$ whose order $|G|=$ $p_{1}^{A_{1}} \cdots p_{r}^{A_{r}}=\prod_{i=1}^{r} p_{i}^{A_{i}}$ can be expressed as

$G \simeq\left(Z_{p_{1}^{a_{1,1}}} \times \cdots \times Z_{p_{1}^{a_{1, n_{1}}}}\right) \times \cdots \times\left(Z_{p_{r}^{a_{r, 1}}} \times \cdots \times Z_{p_{r}^{a_{r, n_{r}}}}\right)$,

where each $p_{i}$ is a distinct prime number and $a_{i, j}$ satisfy

$$
A_{i}=\sum_{j=1}^{n_{i}} a_{i, j}, \quad a_{i, j} \geq a_{i, j+1} .
$$

Note that $a_{i, j}$ is uniquely determined by $G$.

The above theorem uses the following theorem,

The Chinese remainder theorem If $m$ and $n$ are coprime to each other, then

$$
Z_{m n} \simeq Z_{m} \times Z_{n}
$$

is satisfied.

Note that, by using this theorem, the following relation,

$$
Z_{m} \times Z_{n} \simeq Z_{\mathrm{gcd}(m, n)} \times Z_{\mathrm{lcm}(m, n)}
$$

is generally satisfied.
[1] G. Altarelli and F. Feruglio, Rev. Mod. Phys. 82, 2701 (2010).

[2] H. Ishimori, T. Kobayashi, H. Ohki, Y. Shimizu, H. Okada, and M. Tanimoto, Prog. Theor. Phys. Suppl. 183, 1 (2010).

[3] H. Ishimori, T. Kobayashi, H. Ohki, H. Okada, Y. Shimizu, and M. Tanimoto, Lect. Notes Phys. 858, 1 (2012).

[4] D. Hernandez and A. Y. Smirnov, Phys. Rev. D 86, 053014 (2012).

[5] S. F. King and C. Luhn, Rep. Prog. Phys. 76, 056201 (2013).

[6] S. F. King, A. Merle, S. Morisi, Y. Shimizu, and M. Tanimoto, New J. Phys. 16, 045018 (2014).

[7] K. Fujikawa, Phys. Rev. Lett. 42, 1195 (1979).

[8] K. Fujikawa, Phys. Rev. D 21, 2848 (1980); 22, 1499(E) (1980).

[9] L. M. Krauss and F. Wilczek, Phys. Rev. Lett. 62, 1221 (1989).

[10] L. E. Ibanez and G. G. Ross, Phys. Lett. B 260, 291 (1991).

[11] T. Banks and M. Dine, Phys. Rev. D 45, 1424 (1992).
[12] T. Araki, T. Kobayashi, J. Kubo, S. Ramos-Sanchez, M. Ratz, and P. K. S. Vaudrevange, Nucl. Phys. B805, 124 (2008).

[13] M. C. Chen, M. Fallbacher, M. Ratz, A. Trautner, and P. K. S. Vaudrevange, Phys. Lett. B 747, 22 (2015).

[14] L. Alvarez-Gaume and E. Witten, Nucl. Phys. B234, 269 (1984).

[15] L. Alvarez-Gaume and P. H. Ginsparg, Ann. Phys. (N.Y.) 161, 423 (1985); 171, 233(E) (1986).

[16] R. Blumenhagen, B. Kors, D. Lust, and S. Stieberger, Phys. Rep. 445, 1 (2007).

[17] L. E. Ibanez and A. M. Uranga, String Theory and Particle Physics: An Introduction to String Phenomenology (Cambridge University Press, Cambridge, England, 2012).

[18] Y. Hamada, T. Kobayashi, and S. Uemura, J. High Energy Phys. 05 (2014) 116.

[19] Y. Kariyazono, T. Kobayashi, S. Takada, S. Tamba, and H. Uchida, Phys. Rev. D 100, 045014 (2019). 